\title{
THE VOLUME OF TUBES IN COMPLEX PROJECTIVE SPACE $\left({ }^{1}\right)$
}

\author{
BY \\ ROBERT A. WOLF
}

\begin{abstract}
A formula for the volume of a tube about a compact complex submanifold of complex projective space is derived.
\end{abstract}

1. Introduction. If $M^{k}$ is a compact $k$-dimensional submanifold (without boundary) of $n$-dimensional euclidean space $\boldsymbol{R}^{n}$, Weyl's formula [5] for the volume of the tube $M(\rho)$ of radius $\rho$ about $M$ is

$$
\operatorname{vol}(M(\rho))=O_{m} \sum_{0 \leqq e \leqq k: e \text { even }} \frac{\rho^{m+e}}{m(m+2) \cdots(m+e)} k_{e},
$$

where $m=n-k$, where, in general, for $j \geqq 1, O_{j}=2 \pi^{j / 2} / \Gamma(j / 2)$ is the volume of the unit sphere $S^{j-1}$ in euclidean $j$-space, and where $k_{e}$ is the integral over $M$ of the invariant

$$
H_{e}=\frac{1}{4^{e / 2}(e / 2) !} \sum \delta\left(\begin{array}{c}
\alpha_{1} \cdots \alpha_{e} \\
\beta_{1} \cdots \beta_{e}
\end{array}\right) R_{\beta_{1} \beta_{2}}^{\alpha_{1} \alpha_{2}} \cdots R_{\beta_{e}-1 \beta_{e}}^{\alpha_{e}-1 \alpha_{e}}
$$

$R_{y \delta}^{\alpha \beta}$ being the curvature tensor of $M$. Here $\delta\left(\begin{array}{c}\alpha_{1} \ldots \alpha_{e} \cdots \alpha_{e} \\ \beta_{1} \cdots \beta_{e}\end{array}\right)$ is +1 or -1 according as $\alpha_{1}, \ldots, \alpha_{e}$ are distinct and an even or odd permutation of $\beta_{1}, \ldots, \beta_{e}$, and otherwise $\delta\left(\begin{array}{c}\alpha_{1} \cdots \alpha_{e} \\ \beta_{1} \cdots \beta_{e}\end{array}\right)$ is zero. The summation in $H_{e}$ is taken over all $\alpha$ 's and $\beta$ 's running from 1 to $k$. If $M^{k}$ is a compact $k$-dimensional submanifold (without boundary) of the unit sphere $S^{n}$, Weyl's formula for the volume of the tube $M(\rho)$ of radius $\rho$ about $M$ is

$$
\operatorname{vol}(M(\rho))=O_{m} \sum_{0 \leqq e \leqq k ; e \text { even }} \frac{k_{e}}{m(m+2) \cdots(m+e-2)} J_{e}(\rho),
$$

where $m=n-k$ and

$$
J_{e}(\rho)=\int_{0}^{\tan \rho} \frac{r^{e+m-1}}{\left(1+r^{2}\right)^{(n+1) / 2}} d r=\int_{0}^{\rho}(\sin s)^{m+e-1}(\cos s)^{k-e} d s
$$

Received by the editors September 12, 1969 and, in revised form, June 21, 1970. AMS 1969 subject classifications. Primary 5390.

Key words and phrases. Tubes, volume, complex projective space, complex submanifold, holomorphic curvature tensor, second fundamental form.

(1) This paper is a revision of a portion of the author's doctoral dissertation under S.-S. Chern at the University of California at Berkeley. 
and where $k_{e}$ is the integral over $M$ of the invariant

$$
\frac{1}{4^{e / 2}(e / 2) !} \sum \delta\left(\begin{array}{c}
\alpha_{1} \cdots \alpha_{e} \\
\beta_{1} \cdots \beta_{e}
\end{array}\right) H_{\beta_{1} \beta_{2}}^{\alpha_{1} \alpha_{2}} \cdots H_{\beta_{e-1} \beta_{e}}^{\alpha_{e-1} \alpha_{e}}
$$

Here again the $\alpha$ 's and $\beta$ 's run from 1 to $k$, while $H_{\gamma \delta}^{\alpha \beta}$ is the excess curvature tensor of $M$; i.e.,

$$
H_{\gamma \delta}^{\alpha \beta}=R_{\gamma \delta}^{\alpha \beta}-\tilde{R}_{\gamma \delta}^{\alpha \beta}
$$

where $R_{\gamma \delta}^{\alpha \beta}$ is the curvature tensor of $M$ and $\tilde{R}_{\gamma \delta}^{\alpha \beta}$ is that of $S^{n}$.

Now let us consider a compact complex analytic $k$-dimensional submanifold $M^{k}$ of $n$-dimensional complex projective space $\boldsymbol{P}^{n}(\boldsymbol{C})$ supplied with the FubiniStudy metric. Let $R_{\gamma \delta}^{\alpha \beta}$ be the holomorphic curvature tensor of $M$ and let $\widetilde{R}_{\gamma \delta}^{\alpha \beta}$ be that of $\boldsymbol{P}^{n}(\boldsymbol{C})$. Also let

$$
H_{\gamma \delta}^{\alpha \bar{\beta}}=R_{\gamma \delta}^{\alpha \bar{\beta}}-\widetilde{R}_{\gamma \delta}^{\alpha \bar{\beta}}
$$

be the excess holomorphic curvature tensor of $M$. Then on $M$ we have real-valued invariants

$$
H_{2 f}=\sum \delta\left(\begin{array}{l}
\alpha_{1} \cdots \alpha_{f} \\
\gamma_{1} \cdots \gamma_{f}
\end{array}\right) \delta\left(\begin{array}{c}
\beta_{1} \cdots \beta_{f} \\
\delta_{1} \cdots \delta_{f}
\end{array}\right) H_{\gamma_{1} \delta_{1}}^{\alpha_{1} \beta_{1}} \cdots H_{\gamma_{f} \delta_{f}}^{\alpha_{f}^{\beta} \bar{\beta}_{f}}
$$

for $0 \leqq f \leqq k$, where the $\alpha$ 's, $\beta$ 's, $\gamma$ 's, and $\delta$ 's run from 1 to $k$. We shall show that the volume of the tube $M(\rho)$ of all points of $\boldsymbol{P}^{n}(\boldsymbol{C})$ within a distance $\rho$ of $M$ is

$$
\operatorname{vol}(M(\rho))=\sum_{0 \leqq f \leqq k} \frac{2 \pi^{m}}{f !(f+m-1) !} k_{2 f} J_{f}(\rho)
$$

where $m=n-k$, where $k_{2 f}$ is the integral of $H_{2 f}$ over $M$, and where $J_{f}(\rho)$ is defined as

$$
\begin{aligned}
J_{f}(\rho) & =\int_{0}^{\tan \rho} \frac{r^{2 m+2 f-1}}{\left(1+r^{2}\right)^{n+1}} d r \\
& =\int_{0}^{\rho}(\sin s)^{2 m+2 f-1}(\cos s)^{2 k-2 f+1} d s .
\end{aligned}
$$

I wish to thank S.-S. Chern for suggesting this problem.

2. Hermitian manifolds [1], [4]. Let $M$ be a hermitian manifold of complex dimension $n$. For any point $p \in M$, we will let $\left.T_{C}(M)\right|_{p},\left.\left(T_{C}(M)\right)^{-}\right|_{p}$, and $\left.T_{R}(M)\right|_{p}$ denote, respectively, the tangent vectors of type $(1,0)$, the tangent vectors of type $(0,1)$, and the real tangent vectors at $p$. Let $\tilde{\pi}: U(M) \rightarrow M$ be the bundle of unitary frames (of type $(1,0)$ ) defined with respect to the hermitian inner product. On $U(M)$ we have canonical complexified real one-forms (i.e., elements of $\left.\left(T_{R}(U(M)) \times{ }_{R} C\right)^{*}\right) \theta^{1}, \ldots, \theta^{n}$ defined as follows (see [2, p. 110]). 
Pick, in a coordinate neighborhood $V$ of $M, n$ complexified real $C^{\infty}$ one-forms $\varphi^{1}, \ldots, \varphi^{n}$ such that

$$
d s^{2}=\sum_{j=1}^{n} \varphi^{j} \otimes\left(\varphi^{j}\right)^{-}
$$

(here $\left(\varphi^{j}\right)^{-}$is $\varphi^{j}$ followed by the complex conjugation on $C$ ), and then consider the forms

$$
\gamma^{j}=\sum_{k=1}^{n}\left(u^{-1}\right)_{k}^{j} \varphi^{k}, \quad 1 \leqq j \leqq n
$$

defined on $V \times U(n)$, where $\left(u_{k}^{j}\right) \in U(n)$, the group of $n \times n$ unitary matrices. Now, if $f_{1}, \ldots, f_{n}$ are the dual tangent vector fields to the $\varphi$ 's, we get a trivialization $\psi_{f}: \tilde{\pi}^{-1}(V) \rightarrow V \times U(n)$ sending the frame $x\left(\sum u_{1}^{j} f_{j}\right) \cdots\left(\sum u_{n}^{j} f_{j}\right)$ to $\left(x,\left(u_{k}^{j}\right)\right)$, and the pullbacks under $\psi_{f}^{*}$ of the $\gamma$ 's are forms on $\tilde{\pi}^{-1}(V) \subseteq U(M)$. One may check that these forms are independent of the choice of $\varphi$ 's, and they are called the canonical forms $\theta^{1}, \ldots, \theta^{n}$.

For any real manifold $N$ complex conjugation acts on $T_{R}(N) \otimes_{R} C$ and on its dual space (and on their various tensor and exterior products) in a natural way by acting on the " $C$ " in $T_{R}(N) \otimes_{R} C$. This action commutes with tensor product, exterior product, exterior differentiation, and pullbacks. If $\theta$ is a form, $\bar{\theta}$ will be the conjugate. Notice that if $N$ is complex, if $\left(z^{1}, \ldots, z^{n}\right)$ is a local complex coordinate system of $N, n$ being the complex dimension of $N$, and if $x^{j}$ and $y^{j}$ are the real and imaginary parts of $z^{j}, 1 \leqq j \leqq n$, then

$$
\left(d z^{j}\right)^{-}=\left(d x^{j}+i d y^{j}\right)^{-}=d x^{j}-i d y^{j}=d \bar{z}^{j} ;
$$

this notation $\left(d z^{j}\right)^{-}$conflicts with that used in $d s^{2}=\sum g_{j k} d z^{j} \otimes\left(d z^{k}\right)^{-}$for a hermitian manifold $N$, but no confusion will result in our discussion.

For our hermitian manifold $M$, if $\sigma: V \rightarrow U(M)$ is any local $C^{\infty}$ cross section of $U(M)$ over an open subset $V$ of $M$, then the absolute form

$$
\left|(i / 2)^{n} \sigma^{*} \theta^{1} \wedge \sigma^{*} \bar{\theta}^{1} \wedge \cdots \wedge \sigma^{*} \theta^{n} \wedge \sigma^{*} \bar{\theta}^{n}\right|
$$

is the absolute riemannian volume element on $V$, where for $1 \leqq j \leqq n, \bar{\theta}^{j}$ is $\left(\theta^{j}\right)^{-}$, the result of the conjugation operator of the previous paragraph acting on $\theta^{3}$.

There is a unique set of forms $\omega_{k c}^{j} \in\left(T_{R}(U(M)) \otimes_{R} C\right)^{*}, 1 \leqq j, k \leqq n$, satisfying

$$
\omega_{k}^{j}+\bar{\omega}_{j}^{k}=0 \quad\left(\bar{\omega}_{j}^{k} \text { means }\left(\omega_{j}^{k}\right)^{-}\right)
$$

and

$$
d \theta^{j}=-\sum_{k=1}^{n} \omega_{k}^{j} \wedge \theta^{k}+\frac{1}{2} \sum_{k, l=1}^{n} T_{k l}^{j} \theta^{k} \wedge \theta^{l}, \quad 1 \leqq j \leqq n
$$


where $T_{k l}^{j}+T_{l k}^{j}=0,1 \leqq j, k, l \leqq n$ (see [2, p. 111]). These forms are called the connection forms. The equation

$$
d \omega_{k}^{j}=-\sum_{l=1}^{n} \omega_{l}^{j} \wedge \omega_{k}^{l}+\Omega_{k}^{j}, \quad 1 \leqq j, k \leqq n,
$$

is a consequence, with the curvature form $\Omega_{k}^{j}$ necessarily having the form

$$
\Omega_{k}^{j}=\sum_{l, m=1}^{n} R_{k l \bar{m}}^{j} \theta^{l} \wedge \bar{\theta}^{m}, \quad 1 \leqq j, k \leqq n .
$$

Here $R^{j}{ }_{k l m}, 1 \leqq j, k, l, m \leqq n$, is the complex curvature function on $U(M)$ and satisfies $R_{k l \bar{m}}^{j}=R_{l k \bar{m}}^{j}=R^{m}{ }_{k l \bar{j}}=\bar{R}^{k}{ }_{j m i}$. A kähler manifold is defined to be a hermitian manifold whose $T_{k l}^{j}$ 's all vanish identically.

3. The second fundamental form of a complex submanifold of a kähler manifold. Let $M$ be a complex $k$-dimensional submanifold of an $n$-dimensional kähler manifold $N$. Let $U(N, M)$ be the bundle of (adapted) unitary frames $x e_{1} \cdots e_{n}$ satisfying $x \in M, e_{1}, \ldots, e_{k}$ tangent to $M$. So $U(N, M)$ is a principal bundle over $M$ with structure group $U(k) \times U(n-k)$.

Let $\sigma: M \rightarrow N$ and $\sigma: U(N, M) \rightarrow U(N)$ be the inclusion mappings, and let $\pi^{\prime}: U(N, M) \rightarrow U(M)$ be the bundle projection. We will use the following ranges of indices in this section:

$$
1 \leqq i, j, l, m \leqq n ; \quad 1 \leqq \alpha, \beta, \gamma, \delta \leqq k ; \quad k+1 \leqq r, s \leqq n .
$$

Proofs of the following facts are straightforward to supply.

1. The forms $\sigma^{*} \theta^{\alpha}$ on $U(N, M)$ can be regarded as forms on $U(M)$, and in fact, they are the canonical forms on $U(M)$.

2. $\sigma^{*} \theta^{r}=0$ on $U(N, M)$.

3. The forms $\sigma^{*} \omega_{\beta}^{\alpha}$ on $U(N, M)$ can be regarded as forms on $U(M)$, and in fact, they are the connection forms on $U(M)$. Furthermore, on $U(M)$ we have $d \sigma^{*} \theta^{\alpha}$ $=-\sum_{\beta} \sigma^{*} \omega_{\beta}^{\alpha} \wedge \sigma^{*} \theta^{\beta}$. (Hence, $M$ is also kähler with respect to the induced hermitian metric.)

From the equation

$$
d \theta^{r}=-\sum_{\alpha} \omega_{\alpha}^{r} \wedge \theta^{\alpha}-\sum_{s} \omega_{s}^{r} \wedge \theta^{s}
$$

on $U(N)$, we get on $U(N, M)$

$$
0=d \sigma^{*} \theta^{r}=-\sum_{\alpha} \sigma^{*} \omega_{\alpha}^{r} \wedge \sigma^{*} \theta^{\alpha} .
$$

Since the $\sigma^{*} \theta^{\alpha}$ s are pointwise complex linearly independent complexified real 1-forms on $U(N, M)$, we can conclude that there exist unique complex-valued $C^{\infty}$ functions $h_{\alpha \beta}^{r}$ on $U(N, M)$ satisfying $h_{\alpha \beta}^{r}=h_{\beta \alpha}^{r}$ and

$$
\sigma^{*} \omega_{\alpha}^{r}=\sum_{\beta} h_{\alpha \beta}^{r} \sigma^{*} \theta^{\beta}
$$


The $h_{\alpha \beta}^{r}$ are called (the components of) the complex second fundamental form of $M$ in $N$. The $h_{\alpha \beta}^{r}$ transform along the fibers of $U(N, M)$ as tensors. Indices in $h_{\alpha \beta}^{r}$ should acquire or lose a bar when they are raised or lowered. A similar comment holds for $R^{i}{ }_{j l \bar{m}}$.

One more fact we need is that if $X$ is a tangent vector to $U(N, M)$, then $\sigma^{*} \theta^{\alpha}(X)$ and $\sigma^{*} \omega_{\beta}^{\alpha}(X)$ depend only on $\pi_{*}^{\prime} X$. Again, we will omit the proof of this fact. Hence, if $\theta^{\prime \alpha}$ and $\omega_{\beta}^{\prime \alpha}$ temporarily denote $\sigma^{*} \theta^{\alpha}$ and $\sigma^{*} \omega_{\beta}^{\alpha}$ considered on $U(M)$, then $\sigma^{*} \theta^{\alpha}=\left(\pi^{\prime}\right)^{*} \theta^{\prime \alpha}$ and $\sigma^{*} \omega_{\beta}^{\alpha}=\left(\pi^{\prime}\right)^{*} \omega_{\beta}^{\prime \alpha}$ on $U(N, M)$. On $U(M)$ we have

$$
d \omega_{\beta}^{\prime \alpha}+\sum_{\gamma} \omega_{\gamma}^{\prime \alpha} \wedge \omega_{\beta}^{\prime \gamma}=\Omega_{\beta}^{\alpha}=\sum_{\gamma, \delta} R_{\beta \gamma \delta}^{\alpha} \theta^{\prime \gamma} \wedge \tilde{\theta}^{\prime \delta},
$$

the $R^{\alpha}{ }_{\beta \gamma \delta}$ being the curvature functions for $M$. Apply $\left(\pi^{\prime}\right)^{*}$ to this equation to get

$$
d \sigma^{*} \omega_{\beta}^{\alpha}+\sum_{\gamma} \sigma^{*} \omega_{\gamma}^{\alpha} \wedge \sigma^{*} \omega_{\beta}^{\gamma}=\sum_{\gamma, \delta} R_{\beta \gamma \delta}^{\alpha} \circ \pi^{\prime} \sigma^{*} \theta^{\gamma} \wedge \sigma^{*} \bar{\theta}^{\delta}
$$

on $U(N, M)$. But on $U(N, M)$

$$
d \sigma^{*} \omega_{\beta}^{\alpha}+\sum_{\gamma} \sigma^{*} \omega_{\gamma}^{\alpha} \wedge \sigma^{*} \omega_{\beta}^{\gamma}=-\sum_{r} \sigma^{*} \omega_{r}^{\alpha} \wedge \sigma^{*} \omega_{\beta}^{r}+\sum_{\gamma, \delta}\left(\tilde{R}_{\beta \gamma \delta}^{\alpha} \circ \sigma\right) \sigma^{*} \theta^{\gamma} \wedge \sigma^{*} \bar{\theta}^{\delta},
$$

where the $\tilde{R}_{j l \bar{m}}^{i}$ are the curvature functions for $N$. Hence, we get

$$
R_{\beta \gamma \delta}^{\alpha} \circ \pi^{\prime}-\tilde{R}_{\beta \gamma \delta}^{\alpha} \circ \sigma=-\sum_{r} \bar{h}_{\alpha \delta}^{r} h_{\beta \gamma}^{r},
$$

or, as we prefer to write,

$$
R_{\beta \gamma \delta}^{\alpha} \circ \pi^{\prime}-\tilde{R}_{\beta \gamma \delta}^{\alpha} \circ \sigma=-\sum_{r} \bar{h}_{\bar{\gamma} \delta}^{\alpha} h_{\beta \gamma}^{r} .
$$

Raising the index $\beta$, and writing $R_{\gamma \delta}^{\alpha \beta}$ for $R^{\alpha \bar{\beta}}{ }_{\gamma \delta}$, we get

$$
R_{\gamma \delta}^{\alpha \bar{\beta}} \circ \pi^{\prime}-\widetilde{R}_{\gamma \delta}^{\alpha \bar{\beta}} \circ \sigma=-\sum_{r} \bar{h}_{\bar{\tau} \delta}^{\bar{\alpha}} h_{\gamma}^{r \bar{\beta}} .
$$

Since $\widetilde{R}_{\gamma \delta}^{\alpha \bar{\beta}} \circ \sigma$ and $\sum_{r} \bar{h}_{\tilde{r} \delta}^{\alpha} h_{\gamma}^{r \beta}$ do not depend on the last $n-k$ vectors of an adapted unitary frame, they can be considered as $C^{\infty}$ functions on $U(M)$, and we have the fundamental equation

$$
R_{\gamma \delta}^{\alpha \beta}-\tilde{R}_{\gamma \delta}^{\alpha \beta}=-\sum_{r} \bar{h}_{\bar{r} \delta}^{\bar{\alpha}} h_{\gamma}^{r \beta}
$$

on $U(M)$. The quantities $H_{\gamma \delta}^{\alpha \beta}=R_{\gamma \delta}^{\alpha \beta}-\widetilde{R}_{\gamma \delta}^{\alpha \beta}$ are called the components of the excess holomorphic curvature tensor of $M$ in $N$.

4. Comments on complex projective space. In this section indices will have the following ranges:

$$
1 \leqq i, j, k, l, m \leqq n ; \quad 1 \leqq A, B, C \leqq n+1 .
$$


If $z=\left(z^{1}, \ldots, z^{n+1}\right)$ is an $(n+1)$-tuple of complex numbers, not all zero, [z] will be the equivalence class of all such $(n+1)$-tuples which are multiples of one another by nonzero complex numbers. Complex projective $n$-space, $P^{n}(C)$, is the complex $n$-dimensional manifold of such equivalence classes. We let $\pi: C^{n+1}-\{0\} \rightarrow \boldsymbol{P}^{n}(\boldsymbol{C})$ be the natural projection.

Distance in $\boldsymbol{P}^{n}(\boldsymbol{C})$ is defined by the Laguerre formula

$$
\cos \operatorname{dist}([a],[b])=|\langle a, b\rangle| /|a| \cdot|b|,
$$

where $\langle a, b\rangle$ is the inner product of $a$ with $b$ in $C^{n+1}$. Hence, the distance between the points $[a]$ and $[b]$ is the acute angle between the complex lines in $C^{n+1}$ represented by $[a]$ and $[b]$. The infinitesimal version of this distance is the Fubini-Study metric, which is symbolically written

$$
\left.d s^{2}\right|_{[z]}=(\langle d z, d z\rangle\langle z, z\rangle-\langle d z, z\rangle\langle z, d z\rangle) /\langle z, z\rangle^{2},
$$

where $\langle\ldots, \ldots\rangle$ is the inner product in $C^{n+1}$.

The geodesic segment in $\boldsymbol{P}^{n}(\boldsymbol{C})$ from $[a]$ to $[b]$ is given as follows. Multiply $a$ by the appropriate complex number of modulus 1 to insure $\langle a, b\rangle \geqq 0$. Then the geodesic $\gamma$ in question is

$$
\gamma(t)=\left[\frac{a}{|a|} \cos t+\frac{b-a\langle b, a\rangle /|a|^{2}}{\left.|b-a\langle b, a\rangle /| a\right|^{2} \mid} \sin t\right],
$$

for $0 \leqq t \leqq \operatorname{arc} \cos \langle b, a\rangle /(|b| \cdot|a|)$. It lies in the complex line through $[a]$ and $[b]$.

Next, we will provide some details expanding the presentation of the canonical and connection forms on $\boldsymbol{P}^{n}(\boldsymbol{C})$ given in [2]. Let $\varepsilon_{1}, \ldots, \varepsilon_{n+1}$ be the standard basis of $C^{n+1}$. We can identify the unitary group $U(n+1)$ with the manifold of all unitary frames $O f_{1} \cdots f_{n+1}$ based at the origin $O$ in $C^{n+1}$ by means of the mapping which sends the matrix $\left(u_{B}^{A}\right)_{1 \leqq A, B \leqq n+1}$ to the frame $O \sum u_{1}^{A} \varepsilon_{A} \cdots \sum u_{n+1}^{A} \varepsilon_{A}$.

On $U(n+1)$, considered as the space of frames, we have complexified real oneforms $\theta_{A}^{\prime}$ defined by the equation

$$
d f_{A}=\sum_{B} \theta_{A}^{\prime B} \otimes f_{B}
$$

Here $d f_{A}$ means $\sum_{B} d \varepsilon_{A}^{B} \otimes \varepsilon_{B}$, where $\varepsilon_{A}^{B}$ is the function on the space of frames that assigns to a frame $O f_{1} \cdots f_{n+1}$ the $\varepsilon_{B}$ th coordinate of $f_{A}$, where $\varepsilon_{1}, \ldots, \varepsilon_{n+1}$ is the standard basis of $C^{n+1}$.

It follows that

$$
\theta_{A}^{\prime B}=\sum_{C} d \varepsilon_{A}^{C}\left(\varepsilon_{B}^{C}\right)^{-}, \quad \theta_{A}^{B}+\left(\theta_{B}^{\prime}\right)^{-}=0,
$$

and

$$
d \theta_{A}^{\prime B}=-\sum_{C} \theta_{C}^{B} \wedge \theta_{A}^{\prime}
$$


We have principal fiber bundles $p: U(n+1) \rightarrow S^{2 n+1}$ and $\pi: S^{2 n+1} \rightarrow P^{n}(C)$ with fibers $U(n)$ and $S^{1}$, respectively, where $p\left(O f_{1} \cdots f_{n+1}\right)=f_{n+1}$ and $\pi$ is the natural projection. Let $\mu$ be a $C^{\infty}$ local cross section of $\pi p: U(n+1) \rightarrow \boldsymbol{P}^{n}(C)$ over an open subset $V$ of $\boldsymbol{P}^{n}(C)$. Then we also have a map $\mu^{\prime}: V \times U(n) \rightarrow U(n+1)$, where

$$
\mu^{\prime}(x, u)=O\left(\sum_{j} u_{1}^{j} f_{j}\right) \cdots\left(\sum_{j} u_{n}^{j} f_{j}\right) f_{n+1},
$$

where $O f_{1} \cdots f_{n+1}=\mu(x), x \in V$. Then

$$
\left(\mu^{\prime}\right)^{*} \theta_{n+1}^{\prime j}=\left\langle d f_{n+1}, \sum_{k} u_{j}^{k} f_{k}\right\rangle=\sum_{k}\left(u^{-1}\right)_{k}^{j} \mu^{*} \theta_{n+1}^{\prime k} .
$$

Furthermore, the forms $\mu^{*} \theta_{n+1}^{\prime k}$ on $V$ satisfy

$$
d s^{2}=\sum_{k=1}^{n} \mu^{*} \theta_{n+1}^{\prime k} \otimes\left(\mu^{*} \theta_{n+1}^{\prime k}\right)^{-}
$$

(see [2, pp. 116, 117]). Therefore, we know from $\S 2$ that if $e_{1}, \ldots, e_{n}$ are the vector fields on $V$ dual to $\mu^{*} \theta_{n+1}^{\prime 1}, \ldots, \mu^{*} \theta_{n+1}^{\prime n}$ and if $\mu^{\prime \prime}: \tilde{\pi}^{-1}(V) \rightarrow V \times U(n)$ is the trivialization of the bundle $\tilde{\pi}: U\left(\boldsymbol{P}^{n}(\boldsymbol{C})\right) \rightarrow \boldsymbol{P}^{n}(\boldsymbol{C})$ given by

$$
\mu^{\prime \prime}\left(x\left(\sum_{j} u_{1}^{j} e_{j}\right) \cdots\left(\sum_{j} u_{n}^{j} e_{j}\right)\right)=\left(x,\left(u_{k}^{j}\right)\right),
$$

then the canonical forms $\theta^{1}, \ldots, \theta^{n}$ on $U\left(\boldsymbol{P}^{n}(\boldsymbol{C})\right)$ over $V$ are given by

$$
\theta^{j}=\left(\mu^{\prime \prime}\right)^{*}\left(\mu^{\prime}\right)^{*} \theta_{n+1}^{\prime j}
$$

Since the forms $\left(\mu^{\prime}\right) *\left(\theta_{k}^{\prime j}-\delta_{k}^{j} \theta_{n+1}^{\prime n+1}\right)$ satisfy the equations

$$
d\left(\mu^{\prime}\right) * \theta_{n+1}^{\prime j}=-\sum_{k}\left(\mu^{\prime}\right) *\left(\theta_{k}^{\prime j}-\delta_{k}^{j} \theta_{n+1}^{\prime n+1}\right) \wedge\left(\mu^{\prime}\right)^{*} \theta_{n+1}^{\prime k}
$$

on $V \times U(n)$, then the connection forms $\omega_{k}^{j}, 1 \leqq j, k \leqq n$, on $U\left(\boldsymbol{P}^{n}(\boldsymbol{C})\right)$ over $V$ are given by

$$
\omega_{k}^{j}=\left(\mu^{\prime \prime}\right) *\left(\mu^{\prime}\right) *\left(\theta_{k}^{j}-\delta_{k}^{j} \theta_{n+1}^{\prime n+1}\right)
$$

(see [2, pp. 116, 117]).

From the definition of the Fubini-Study metric it follows that $U(n+1)$ is a principal fiber bundle over $U\left(\boldsymbol{P}^{n}(\boldsymbol{C})\right)$ with fiber $S^{1}$ and with projection sending $O f_{1} \cdots f_{n+1}$ to $\left[f_{n+1}\right] \pi_{*}\left(\left.f_{1}\right|_{f_{n+1}}\right) \cdots \pi_{*}\left(\left.f_{n}\right|_{f_{n+1}}\right)$, where $\pi: \boldsymbol{C}^{n+1}-\{0\} \rightarrow \boldsymbol{P}^{n}(\boldsymbol{C})$ is the natural projection and $\left.f_{i}\right|_{f_{n+1}}$ is the tangent vector of type $(1,0)$ at the point $f_{n+1} \in C^{n+1}$ which is the image of $f_{i}$ under the canonical isometry

$$
\left.C^{n+1} \rightarrow T_{C}\left(C^{n+1}\right)\right|_{f_{n+1}} .
$$


LEMMA 4.1. Let $\mu$ be a $C^{\infty}$ local cross section of $U(n+1)$ over an open subset $V$ of $\boldsymbol{P}^{n}(\boldsymbol{C})$, and let $\mu^{\prime}$ and $\mu^{\prime \prime}$ be the maps discussed above that are associated to $\mu$. Then $\mu^{\prime} \circ \mu^{\prime \prime}$ is a $C^{\infty}$ local cross section of $U(n+1)$ over the open subset $\tilde{\pi}^{-1}(V)$ of $U\left(\boldsymbol{P}^{n}(\boldsymbol{C})\right)$.

Proof. Let $e_{1}, \ldots, e_{n}$ be the vector fields on $V$ dual to $\mu^{*} \theta_{n+1}^{\prime 1}, \ldots, \mu^{*} \theta_{n+1}^{\prime n}$. For $x \in V$, write $\mu(x)=O f_{1} \cdots f_{n+1}$. Then we will prove first that $\pi_{*}\left(\left.f_{j}\right|_{f_{n+1}}\right)=e_{j}$. I.e., we will show $\left(\mu^{*} \theta_{n+1}^{i t}\right) \pi_{*}\left(\left.f_{j}\right|_{f_{n+1}}\right)=\delta_{j}^{i}$.

To show this, write $f_{B}=\sum f_{B}^{A} \varepsilon_{A}$. Then by equation (4.1)

$$
\begin{aligned}
\mu^{*} \theta_{n+1}^{\prime i}\left(\pi_{*}\left(\left.f_{j}\right|_{f_{n+1}}\right)\right) & =\left(\sum d \varepsilon_{n+1}^{A}\left(\varepsilon_{i}^{A}\right)^{-}\right)\left(\mu_{*} \pi_{*}\left(\left.f_{j}\right|_{f_{n+1}}\right)\right) \\
& =\sum\left(\left.f_{j}\right|_{f_{n+1}}\left(\varepsilon_{n+1}^{A} \circ \mu \pi\right)\right)\left(f_{i}^{A}\right)^{-} .
\end{aligned}
$$

We know $\left.f_{j}\right|_{f_{n+1}}=\left.\sum f_{j}^{B}\left(\partial / \partial z^{B}\right)\right|_{f_{n+1}}$. Also, there is a complex-valued $C^{\infty}$ function $\eta$ on $\pi^{-1}(V) \subseteq C^{n+1}-\{0\}$ such that for $\left(w^{1}, \ldots, w^{n+1}\right) \in \pi^{-1}(V)$,

$$
\varepsilon_{n+1}^{A} \circ \mu \pi\left(w^{1}, \ldots, w^{n+1}\right)=\eta w^{A} .
$$

Therefore,

$$
\begin{aligned}
\sum_{A}\left(\left.f_{j}\right|_{f_{n+1}}\left(\varepsilon_{n+1}^{A} \circ \mu \pi\right)\right)\left(f_{i}^{A}\right)^{-} & =\left.\sum_{A, B} f_{j}^{B}\left(\frac{\partial \eta}{\partial z^{B}} f_{n+1}^{A}+\eta \delta_{B}^{A}\right)\right|_{f_{n+1}}\left(f_{i}^{A}\right)^{-} \\
& =\left.\sum_{A, B} f_{j}^{B} \frac{\partial \eta}{\partial z^{B}}\right|_{f_{n+1}} f_{n+1}^{A}\left(f_{i}^{A}\right)^{-}+\eta\left(f_{n+1}\right) \sum_{B} f_{j}^{B}\left(f_{i}^{B}\right)^{-} \\
& =0+\eta\left(f_{n+1}\right) \delta_{i j}=\delta_{i j}
\end{aligned}
$$

since $\eta=1$ at $f_{n+1}$. Thus we have proved that $\pi_{*}\left(\left.f_{j}\right|_{f_{n+1}}\right)=e_{j}$.

Now we see that if $\pi_{*}$ also denotes the projection of $U(n+1)$ onto $U\left(\boldsymbol{P}^{n}(\boldsymbol{C})\right)$, then

$$
\begin{aligned}
\pi_{*}\left(\mu^{\prime} \mu^{\prime \prime}\left(x \sum u_{1}^{j} e_{j} \cdots \sum u_{n}^{j} e_{j}\right)\right) & =\pi_{*}\left(O\left(\sum u_{1}^{j} f_{j}\right) \cdots\left(\sum u_{n}^{j} f_{j}\right) f_{n+1}\right) \\
& =x \sum u_{1}^{j} \pi_{*}\left(\left.f_{j}\right|_{f_{n+1}}\right) \cdots \sum u_{n}^{j} \pi_{*}\left(\left.f_{j}\right|_{f_{n+1}}\right) \\
& =x \sum u_{1}^{j} e_{j} \cdots \sum u_{n}^{j} e_{j} .
\end{aligned}
$$

Thus $\mu^{\prime} \mu^{\prime \prime}$ is a local cross section of $U(n+1)$. Q.E.D.

So the canonical forms on $U\left(\boldsymbol{P}^{n}(\boldsymbol{C})\right)$ are obtained as the pullbacks of the $\theta_{n+1}^{\prime j}$ on $U(n+1)$ by means of the local cross sections $\mu^{\prime} \mu^{\prime \prime}$. From this fact and the following lemma it follows that the canonical forms are obtained as the pullbacks of the $\theta_{n+1}^{\prime j}$ by means of any $C^{\infty}$ local cross sections whatsoever.

LEMMA 4.2. If $\lambda_{1}$ and $\lambda_{2}$ are two $C^{\infty}$ local cross sections of $U(n+1)$ over the open subset $W$ of $U\left(\boldsymbol{P}^{n}(\boldsymbol{C})\right)$, then $\lambda_{1} * \theta_{n+1}^{\prime j}=\lambda_{2} * \theta_{n+1}^{\prime j}$ on $W$. 
Proof. If $f$ is a point of $C^{n+1}-\{0\}$ and if $\rho$ is a nonzero complex number, it is easily checked that $\pi_{*}\left(\left.\left(\partial / \partial z^{A}\right)\right|_{\rho f}\right)=\rho^{-1} \pi_{*}\left(\left.\left(\partial / \partial z^{A}\right)\right|_{f}\right)$. Therefore, there is a complexvalued $C^{\infty}$ function $\rho$ of modulus one on $W$ such that if $\lambda_{1}\left(x e_{1} \cdots e_{n}\right)=O f_{1} \cdots f_{n} f_{n+1}$, then $\lambda_{2}\left(x e_{1} \cdots e_{n}\right)=O \rho f_{1} \cdots \rho f_{n} \rho f_{n+1}$. Consequently, $\lambda_{1}^{*} \theta_{n+1}^{\prime j}=\left\langle d f_{n+1}, f_{j}\right\rangle$ while

$$
\begin{aligned}
\lambda_{2}^{*} \theta_{n+1}^{\prime j} & =\left\langle d\left(\rho f_{n+1}\right), \rho f_{j}\right\rangle=\left\langle(d \rho) f_{n+1}+\rho d f_{n+1}, \rho f_{j}\right\rangle \\
& =\left\langle d f_{n+1}, f_{j}\right\rangle=\lambda_{1}^{*} \theta_{n+1}^{\prime j} .
\end{aligned}
$$

Similarly, we have

LEMMA 4.3. $\lambda_{1}^{*} \theta_{j}^{\prime i}=\lambda_{2}^{*} \theta_{j}^{\prime i}$ if $i \neq j$.

Finally we remark that a straightforward calculation shows that the curvature form on $U\left(\boldsymbol{P}^{n}(\boldsymbol{C})\right)$ is given by

$$
\Omega_{j}^{i}=\theta^{i} \wedge \bar{\theta}^{j}+\delta_{j}^{i} \sum_{l} \theta^{l} \wedge \bar{\theta}^{l} .
$$

Hence, the holomorphic curvature function on $U\left(\boldsymbol{P}^{n}(\boldsymbol{C})\right)$ is

$$
R^{i l \bar{m}}=\delta_{i j} \delta_{l m}+\delta_{i l} \delta_{j m}
$$

5. The bundle $F$ for a submanifold of $P^{n}(C)$. Let $M$ be a compact complex submanifold of complex dimension $k$ imbedded in $\boldsymbol{P}^{n}(\boldsymbol{C})$. Henceforth, we let $m=n-k$. Also, for the remainder of this paper indices will have the following ranges: $1 \leqq \alpha, \beta, \gamma, \delta \leqq k ; k+1 \leqq r, s \leqq n ; 1 \leqq A, B, C \leqq n+1 ; 1 \leqq i, j \leqq n$.

Let $F$ be the bundle over $U\left(\boldsymbol{P}^{n}(\boldsymbol{C}), M\right)$ induced by the bundle $U(n+1) \rightarrow U\left(\boldsymbol{P}^{n}(\boldsymbol{C})\right)$ of $\S 4$. So $F$ consists of all $O f_{1} \cdots f_{n+1}$ in $U(n+1)$ such that $\pi\left(f_{n+1}\right) \in M$ and $\pi_{*}\left(\left.f_{1}\right|_{f_{n+1}}\right), \ldots, \pi_{*}\left(\left.f_{k}\right|_{f_{n+1}}\right)$ are tangent to $M$. It is a bundle with fiber $S^{1}$ over $U\left(\boldsymbol{P}^{n}(\boldsymbol{C}), M\right)$. Consisting of frames in $C^{n+1}, F$ will serve as a convenient substitute for $U\left(\boldsymbol{P}^{n}(\boldsymbol{C}), M\right)$.

By means of the inclusion mapping $\tau$ of $F$ into $U(n+1)$ we pull back the forms $\theta_{A}^{\prime}{ }_{A}$ from $U(n+1)$ to $F$. We drop the prime sign and denote the forms on $F$ by $\theta_{A}^{B}$.

Proposition 5.1. $\theta_{n+1}^{r}=0$ on $F$.

Proof. Consider $F$ as a fiber bundle over $M$. Let $\lambda: V \rightarrow F$ be a $C^{\infty}$ local cross section of $F$ over an open subset $V$ of $M$, and let $X$ be a real tangent vector to $M$ at $[w] \in V$. We claim

$$
\left.\sum \theta_{n+1}^{A}\left(\lambda_{*} X\right) f_{A}(\lambda([w]))\right|_{f_{n+1}(\lambda([w]))}=\left(f_{n+1}\right)_{*} \lambda_{*} X,
$$

where $f_{A}$ is the map $f_{A}: F \rightarrow C^{n+1}$ which sends a frame into its $A$ th vector, and where $\left(f_{n+1}\right)_{*}$ is the map on tangent vectors to $F$ induced by the map $f_{n+1}: F \rightarrow C^{n+1}$.

To see this, let $\varepsilon^{1}, \ldots, \varepsilon^{n+1}$ be the dual basis of the standard basis of $C^{n+1}$, and apply both sides of equation (5.1) to $\sum_{C}\left(f_{B}^{C}\right)^{-} \varepsilon^{C}$, the dual basis of $\left.f_{A}\right|_{f_{n+1}(\lambda([w])) \text {, }}$ 
where we write $f_{A}=f_{A}(\lambda([w]))$ and $f_{A}=\sum f_{A}^{B} \varepsilon_{B}$ in terms of the standard basis $\varepsilon_{B}$ of $C^{n+1}$; in the verification, make use of equation (4.1) and the equation $\varepsilon^{B} \circ f_{n+1}$ $=\varepsilon_{n+1}^{B} \circ \tau$.

Apply $\pi_{*}$ to equation (5.1) and get

$$
\left.\sum \theta_{n+1}^{A}\left(\lambda_{*} X\right) \pi_{*} f_{A}\right|_{f_{n+1}}=\pi_{*}\left(f_{n+1}\right)_{*} \lambda_{*} X=X .
$$

Now, $X$ is tangent to $M, \pi_{*} f_{n+1}=0$, the real and imaginary parts of the $\pi_{*} f_{i}$ constitute $2 n$ linearly independent vectors, and the real and imaginary parts of the $\pi_{*} f_{r}$ are normal to $M$. Consequently, $\theta_{n+1}^{r}\left(\lambda_{*} X\right)=0$.

Let us next consider a real tangent vector $Y$ to the fiber of $F$ over $[w]$. Suppose $Y$ is tangent at the frame $O f_{1}^{0} \cdots f_{n+1}^{0}$. Then $Y$ equals $\dot{\gamma}(0)$ for some curve $\gamma(t)$ in the fiber, and we have $f_{n+1}(\gamma(t))=\eta(t) f_{n+1}^{0}$, for a complex-valued $C^{\infty}$ function $\eta$ of modulus one, and $\gamma(0)=O f_{1}^{0} \cdots f_{n+1}^{0}$. Clearly, the tangent vector

$$
\left.\sum \theta_{n+1}^{A}(Y) f_{A}^{0}\right|_{f_{n+1}^{0}}=\left(f_{n+1}\right)_{*} Y
$$

has no component along the $\left.f_{i}^{0}\right|_{f_{n+1}^{0}}$ 's. Hence, $\theta_{n+1}^{i}(Y)=0$. In particular, $\theta_{n+1}^{r}(Y)$ $=0$.

Finally, since any real tangent vector to $F$ is of the form $\lambda_{*} X+Y$, for some real tangent vector $X$ to $M, C^{\infty}$ local cross section $\lambda$ of $F$ over $M$, and real tangent vector $Y$ to the fiber of $F$ over $M$, we must have $\theta_{n+1}^{r}$ vanishing identically on $F$. Q.E.D.

Consequently, on $F$ we have

$$
0=d \theta_{n+1}^{r}=-\sum_{A} \theta_{A}^{r} \wedge \theta_{n+1}^{A}=-\sum_{\alpha} \theta_{\alpha}^{r} \wedge \theta_{n+1}^{\alpha} .
$$

Lemma 5.2. If $\gamma$ is a $C^{\infty}$ local cross section of $F$ over an open subset $W$ of $U\left(\boldsymbol{P}^{n}(\boldsymbol{C}), M\right)$, if $\theta^{i}$ and $\omega_{j}^{i}$ are the canonical and connection forms on $U\left(\boldsymbol{P}^{n}(\boldsymbol{C})\right)$, and if $\sigma: U\left(\boldsymbol{P}^{n}(\boldsymbol{C}), M\right) \rightarrow U\left(\boldsymbol{P}^{n}(\boldsymbol{C})\right)$ is the inclusion, then $\gamma^{*} \theta_{n+1}^{\beta}=\sigma^{*} \theta^{\beta}$ and $\gamma^{*} \theta_{\alpha}^{r}$ $=\sigma^{*} \omega_{\alpha}^{r}$.

Proof. As before, let $\tau: F \rightarrow U(n+1)$ be the inclusion. Shrinking $W$, if necessary, we can extend $\tau \circ \gamma$ to a $C^{\infty}$ local cross section $\lambda$ of $U(n+1)$ over an open subset of $U\left(\boldsymbol{P}^{n}(\boldsymbol{C})\right)$. Then $\tau \circ \gamma=\lambda \circ \sigma$. By equations (4.3) and (4.4) and Lemmas 4.1, 4.2, and 4.3, we get $\lambda^{*} \theta_{n+1}^{\prime \beta}=\theta^{\beta}$ and $\lambda^{*} \theta_{\alpha}^{\prime r}=\omega_{\alpha}^{r}$. The desired result now follows. Q.E.D.

Consequently, since the forms $\sigma^{*} \theta^{\beta}$ are pointwise complex linearly independent on $U\left(\boldsymbol{P}^{n}(\boldsymbol{C}), M\right)$, the forms $\theta_{n+1}^{\beta}$ must be pointwise complex linearly independent on $F$. Therefore, it follows from equation (5.2) that there exist unique complexvalued $C^{\infty}$ functions $b_{\alpha \beta}^{r}$ on $F$ such that $b_{\alpha \beta}^{r}=b_{\beta \alpha}^{r}$ and

$$
\theta_{\alpha}^{r}=\sum b_{\alpha \beta}^{r} \theta_{n+1}^{\beta} .
$$


Then

$$
\theta_{r}^{\alpha}=-\sum \bar{b}_{\alpha \beta}^{r} \bar{\theta}_{n+1}^{\beta},
$$

too. We will usually write $b_{\bar{r} \beta}^{\bar{\alpha}}$ for $b_{\alpha \beta}^{r}$, and $\bar{b}_{\bar{r} \beta}^{\bar{\alpha}}$ for $\bar{b}_{\alpha \beta}^{r}$.

Also we remark that from Lemma 5.2, equation (3.1), and equation (5.3) it follows that if $\gamma$ is a $C^{\infty}$ local cross section of $F$ over an open subset $W$ of $U\left(\boldsymbol{P}^{n}(\boldsymbol{C}), M\right)$, then

$$
b_{\alpha \beta}^{r} \circ \gamma=h_{\alpha \beta}^{r}
$$

on $W$.

Proposition 5.3. Let $\lambda$ be a $C^{\infty}$ local cross section of $F$ over an open subset $U$ of $M$. Let $\sigma: M \rightarrow \boldsymbol{P}^{n}(\boldsymbol{C})$ be the inclusion. If $d s^{2}$ is the Fubini-Study metric on $\boldsymbol{P}^{n}(\boldsymbol{C})$, then on $U$ the metric $\sigma^{*} d s^{2}$ of $M$ is given by

$$
\sigma^{*} d s^{2}=\sum \lambda^{*} \theta_{n+1}^{\alpha} \otimes\left(\lambda^{*} \theta_{n+1}^{\alpha}\right)^{-} .
$$

Proof. Letting $\tau: F \rightarrow U(n+1)$ be the inclusion and shrinking $U$ if necessary, we can extend $\tau \circ \lambda$ to a $C^{\infty}$ local cross section $\mu$ of $U(n+1)$ over an open subset of $\boldsymbol{P}^{n}(\boldsymbol{C})$. So locally $\tau \circ \lambda=\mu \circ \sigma$. The proposition now follows from equation (4.2) and Proposition 5.1. Q.E.D.

Finally we remark that since the riemannian metric induced by $\sigma^{*} d s^{2}$ is positive definite, it follows from Proposition 5.3 that the real and imaginary parts of all the forms $\lambda^{*} \theta_{n+1}^{\alpha}, 1 \leqq \alpha \leqq k$, constitute a collection of $2 k$ pointwise complex linearly independent forms, and from this it follows that the collection

$$
\left\{\lambda^{*} \theta_{n+1}^{\alpha}, \lambda^{*} \bar{\theta}_{n+1}^{\alpha}, 1 \leqq \alpha \leqq k\right\}
$$

consists of $2 k$ pointwise complex linearly independent forms.

6. Tubes about $M$. Let $N(M)$ be the manifold of all real tangent vectors to $\boldsymbol{P}^{n}(\boldsymbol{C})$ that are normal to $M$. For $x \in M$ let $N_{x}(M)$ be the real vector space of real tangent vectors to $\boldsymbol{P}^{n}(C)$ at $x$ normal to $M$. For $\rho>0$ let $B_{x}(\rho)$ be the open ball about the origin in $N_{x}(M)$ consisting of vectors of length less than $\rho$. Since $M$ is compact, there is a positive number $b \leqq \pi / 2$ such that for any positive $\rho \leqq b$, the exponential map exp maps $\bigcup_{x \in M} B_{x}(\rho) \subseteq N(M)$ diffeomorphically onto an open submanifold of $\boldsymbol{P}^{n}(\boldsymbol{C})$ such that dist $(x, \exp X)=\|X\|$, for all $x \in M$ and $X \in B_{x}(\rho)$, where $\|\cdots\|$ is the norm on $N_{x}(M)$. So $\exp \left(\bigcup_{x \in M} B_{x}(\rho)\right)$ is the tube about $M$ consisting of all points of $\boldsymbol{P}^{n}(\boldsymbol{C})$ at a distance less than $\rho$ from $M$. We denote this tube by $M(\rho)$.

For any nonnegative integer $p$ and for any $c>0$, define $B^{p}(c) \subseteq C^{p}$ to be $\left\{\left(z^{1}, \ldots, z^{p}\right): \sum\left|z^{j}\right|^{2}<c^{2}\right\}$. Given any $C^{\infty}$ local cross section $\lambda$ of $F$ over an open subset $U$ of $M$, we can consider the associated map

$$
\lambda^{\prime}: U \times B^{m}(\tan \rho) \rightarrow \exp \left(\bigcup_{x \in U} B_{x}(\rho)\right)
$$


defined by

$$
\lambda^{\prime}\left(x,\left(z^{k+1}, \ldots, z^{n}\right)\right)=\left[f_{n+1}+\sum z^{r} f_{r}\right]
$$

Here $m=n-k$ and $O f_{1} \cdots f_{n+1}$ is $\lambda(x)$.

Proposition 6.1. $\lambda^{\prime}$ is a diffeomorphism of $U \times B^{m}(\tan \rho)$ onto $\exp \left(\bigcup_{x \in U} B_{x}(\rho)\right)$.

Proof. $\lambda^{\prime}\left(U \times B^{m}(\tan \rho)\right) \subseteq \exp \left(\bigcup_{x \in U} B_{x}(\rho)\right)=M(\rho)$ since, when $\sum\left|z^{r}\right|^{2}<\tan ^{2} \rho$,

$$
\cos \operatorname{dist}\left(\left[f_{n+1}\right],\left[f_{n+1}+\sum z^{r} f_{r}\right]\right)=\left(1+\sum\left|z^{r}\right|^{2}\right)^{-1 / 2}>\cos \rho \text {. }
$$

So

$$
\operatorname{dist}\left(x, \lambda^{\prime}\left(x,\left(z^{k+1}, \ldots, z^{n}\right)\right)\right)=\operatorname{dist}\left(\left[f_{n+1}\right],\left[f_{n+1}+\sum z^{r} f_{r}\right]\right)<\rho .
$$

Next we show $\lambda^{\prime}$ is one-to-one on $U \times B^{m}(\tan \rho)$. If

$$
q=\left[f_{n+1}(x)+\sum z^{r} f_{r}(x)\right]=\left[f_{n+1}(y)+\sum w^{r} f_{r}(y)\right]
$$

is the image under $\lambda^{\prime}$ of both $\left(x,\left(z^{r}\right)\right)$ and $\left(y,\left(w^{r}\right)\right)$, then $q$ lies on both the complex line in $\boldsymbol{P}^{n}(\boldsymbol{C})$ joining $x$ to $q$ and on the complex line in $\boldsymbol{P}^{n}(\boldsymbol{C})$ joining $y$ to $q$. Thus there are two real geodesic segments of lengths less than $\rho$, one from $x$ to $q$ lying in the complex line joining $x$ and $q$, the other from $y$ to $q$ lying in the complex line joining $y$ and $q$. Since $\pi_{*}\left(\left.\sum z^{r} f_{r}(x)\right|_{f_{n+1}(x)}\right)$ and $\pi_{*}\left(\left.\sum w^{r} f_{r}(y)\right|_{f_{n+1}(y)}\right)$ are tangents of type $(1,0)$ at $x$ and $y$ to these complex lines, respectively, and since these tangents are normal to $M$, the two complex lines emanate normally from $M$. Hence, the real geodesic segments emanate normally from $M$. But exp is one-to-one on $\bigcup_{x \in M} B_{x}(\rho)$, so the two geodesics must coincide. Hence, $x=y$ and $z^{r}=w^{r}$, and $\lambda^{\prime}$ is one-to-one.

Next we will show $\lambda^{\prime}$ maps $U \times B^{m}(\tan \rho)$ onto $\exp \left(\bigcup_{x \in U} B_{x}(\rho)\right)$. We will use the fact that if $K$ is a compact submanifold of a riemannian manifold $N$, if $p$ is a point of $N-K$, and if $\gamma$ is a geodesic segment from $K$ to $p$ whose length is the distance of $p$ from $K$, then $\gamma$ emanates normally from $K$. Now let $p$ be a point of $\exp \left(\bigcup_{x \in U} B_{x}(\rho)\right)-M$. Let $x_{p}$ be the point in $M$ nearest to $p$. If we let $O f_{1} \cdots f_{n+1}$ $=\lambda\left(x_{p}\right)$, then we can write

$$
p=\left[f_{n+1}+\sum z^{\alpha} f_{\alpha}+\sum z^{r} f_{r}\right]
$$

for some $z$ 's; we know that the coefficient of $f_{n+1}$ is indeed nonzero since, otherwise, $\operatorname{dist}\left(p,\left[f_{n+1}\right]\right)=\pi / 2$, whereas in actuality all points of our tube are at a distance less than $\pi / 2$ from $M$. We next claim that all the $z^{\alpha}$ 's vanish. For suppose not. Then $\pi_{*}\left(\left.\left(\sum z^{\alpha} f_{\alpha}+\sum z^{r} f_{r}\right)\right|_{f_{n+1}}\right)$ is a vector of type $(1,0)$ at $x_{p}$ to the complex line joining $x_{p}$ to $p$, and is clearly not normal to $M$. Hence, if $X$ is any nonzero real tangent to this complex line at $x_{p}, X$ cannot be normal to $M$; for if it were, then so would $J X$ be normal to $M$, and then $X-i J X$ would be a nonzero vector of type $(1,0)$ tangent at $x_{p}$ to the complex line from. $x_{p}$ to $p$ and yet normal to $M$. Finally, 
if $\gamma_{p}$ is the real minimizing geodesic from $x_{p}$ to $p$, then $\gamma_{p}$ lies in this complex line; but the tangent to $\gamma_{p}$ at $x_{p}$ cannot be normal to $M$. This contradiction shows that the $z^{\alpha}$ 's are zero. Thus $p=\left[f_{n+1}+\sum z^{r} f_{r}\right]$, and $0<\sum\left|z^{r}\right|^{2}<\tan ^{2} \rho$ since $p \in M(\rho)-M$. Hence, $p=\lambda^{\prime}\left(x_{p}\right)$ and $\lambda^{\prime}$ is onto.

Since $\lambda^{\prime}$ is clearly $C^{\infty}$, we have only to show $\left(\lambda^{\prime}\right)^{-1}$ is $C^{\infty}$. Let $p$ be a point of $\exp \left(\bigcup_{x \in U} B_{x}(\rho)\right)$ and let $x_{p}$ be the point in $M$ nearest to $p$. Let $O f_{1} \cdots f_{n+1}=\lambda\left(x_{p}\right)$. Then $p=\left[f_{n+1}+\sum z^{r} f_{r}\right]$, for some $z^{r}$ s, and since $x_{p}$ depends on $p$ in a $C^{\infty}$ fashion and since $\lambda$ is $C^{\infty}$, then the map $p \rightarrow f_{n+1}+\sum z^{r} f_{r}$ is $C^{\infty}$, and in fact, the real and imaginary parts of the $z^{r}$ 's are $C^{\infty}$ functions of $p$. Hence $\left(\lambda^{\prime}\right)^{-1}$ is $C^{\infty}$. Q.E.D.

7. The volume element of a tube. We will deal with multivectors having exterior differential forms as coefficients (see [3]). Let $y:\left.C^{n+1} \rightarrow T_{C}\left(C^{n+1}\right)\right|_{0}$ be the natural identification. So, at $\left(z^{1}, \ldots, z^{n+1}\right)$,

$$
y=\sum z^{A}\left(\left.\varepsilon_{A}\right|_{0}\right)
$$

where $\varepsilon_{1}, \ldots, \varepsilon_{n+1}$ is the canonical basis of $C^{n+1}$. Also,

$$
\bar{y}=\sum \bar{z}^{A}\left(\left.\varepsilon_{A}\right|_{0}\right)^{-} \text {. }
$$

It is important to observe that $\left(\left.\varepsilon_{A}\right|_{0}\right)^{-}=\partial /\left.\partial \bar{z}^{A}\right|_{0}$, whereas $\left.\left(\varepsilon_{A}\right)^{-}\right|_{0}=\left.\varepsilon_{A}\right|_{0}=\partial /\left.\partial z^{A}\right|_{0}$.

The form $d y$ is the canonical vector-valued one-form in $C^{n+1}$; i.e., at $\left(z^{1}, \ldots, z^{n+1}\right)$,

$$
d y=\sum d z^{A} \otimes\left(\left.\varepsilon_{A}\right|_{0}\right)
$$

in $\left.\left.T_{C}\left(C^{n+1}\right)\right|_{\left(z^{1}, \ldots, z^{n+1}\right)} ^{\theta} \otimes_{C} T_{C}\left(C^{n+1}\right)\right|_{0}$. Also

$$
(d y)^{-}=d \bar{y}=\sum d \bar{z}^{A} \otimes\left(\left.\varepsilon_{A}\right|_{0}\right)^{-} \text {. }
$$

Now consider the map $\varphi: F \times B^{m}(\tan \rho) \rightarrow C^{n+1}$ given by

$$
\varphi\left(O f_{1} \cdots f_{n+1},\left(z^{k+1}, \ldots, z^{n}\right)\right)=f_{n+1}+\sum z^{r} f_{r},
$$

the map $v: C^{n+1}-\{0\} \rightarrow S^{2 n+1}$ which sends each $v \in C^{n+1}-\{0\}$ into $v /|v|$, and the map $j: S^{2 n+1} \rightarrow C^{n+1}$ which is the inclusion. Then $\varphi^{*} \nu^{*} j^{*} d y$ is an element of

$$
\left.\left(T_{R}\left(F \times B^{m}(\tan \rho)\right) \otimes_{R} C\right)^{*} \otimes_{C} T_{C}\left(C^{n+1}\right)\right|_{0} .
$$

In shorthand, $d(y /|y|)$ stands for $\varphi^{*} \nu^{*} j^{*} d y$ where $y=f_{n+1}+\sum z^{r} f_{r}$, which is identified with $\left.f_{n+1}\right|_{0}+\left.\sum z^{r} f_{r}\right|_{0}$. Also, $d(\bar{y}|| y \mid)$ stands for $\varphi^{*} \nu^{*} j^{*} d \bar{y}$ where

$$
\bar{y}=\sum\left(\left(f_{n+1}^{A}\right)^{-}+\sum \bar{z}^{r}\left(f_{r}^{A}\right)^{-}\right)\left(\left.\varepsilon_{A}\right|_{0}\right)^{-}=\left(\left.f_{n+1}\right|_{0}\right)^{-}+\sum \bar{z}^{r}\left(\left.f_{r}\right|_{0}\right)^{-} .
$$

In the calculations below we will use this shorthand, and we will write $f_{A}$ and $\left(f_{A}\right)^{-}$for $\left.f_{A}\right|_{0}$ and $\left(\left.f_{A}\right|_{0}\right)^{-}$, respectively. We will use the equations $d f_{A}=\sum \theta_{A}^{B} f_{B}$ and $d\left(f_{A}\right)^{-}=\sum \bar{\theta}_{A}^{B} \bar{f}_{B}$.

We now calculate that 
360

R. A. WOLF

[June

$$
\begin{aligned}
d\left(\frac{y}{|y|}\right)= & \frac{1}{|y|} d y-\frac{y}{|y|^{2}} d|y| \\
= & \frac{1}{\left(1+\sum\left|z^{r}\right|^{2}\right)^{1 / 2}}\left\{\sum_{\alpha}\left(\theta_{n+1}^{\alpha}-\sum_{r, \beta} z^{r} \bar{b}_{\tilde{r} \bar{\beta}}^{\alpha} \bar{\theta}_{n+1}^{\beta}\right) f_{\alpha}\right. \\
& \left.\quad+\sum_{s}\left(d z^{s}+\sum_{r} z^{r} \theta_{r}^{s}\right) f_{s}+\theta_{n+1}^{n+1} f_{n+1}\right\} \\
& \quad-\frac{1 / 2}{\left(1+\sum\left|z^{r}\right|^{2}\right)^{3 / 2}} \sum_{r}\left(d z^{r} \bar{z}^{r}+z^{r} d \bar{z}^{r}\right)\left(f_{n+1}+\sum_{s} z^{s} f_{s}\right) .
\end{aligned}
$$

Thus, $[d(y /|y|)]^{n} \wedge[d(\bar{y} /|y|)]^{n} \wedge y /|y| \wedge \bar{y} /|y|$, which is shorthand for

$$
\varphi^{*} \nu^{*} j^{*}\left[(d y)^{n} \wedge(d \bar{y})^{n} \wedge y \wedge \bar{y}\right]
$$

is

$$
\begin{aligned}
& \frac{y \wedge \bar{y}}{|y|^{2 n+2}}\left\{\left(\begin{array}{l}
n \\
k
\end{array}\right)^{2}\left[\sum_{\alpha}\left(\theta_{n+1}^{\alpha}-\sum_{r, \beta} z^{r} \bar{b}_{\tilde{r} \boldsymbol{\alpha}}^{\bar{\alpha}} \bar{\theta}_{n+1}^{\beta}\right) f_{\alpha}\right]^{k} \wedge\left[\sum_{s}\left(d z^{s}+\sum_{r} z^{r} \theta_{r}^{s}\right) f_{s}\right]^{n-k}\right. \\
& \wedge\left[\left(\sum_{\alpha}\left(\theta_{n+1}^{\alpha}-\sum_{r, \beta} z^{r} \bar{b}_{\bar{r} \tilde{\beta}}^{\bar{\alpha} \theta_{n+1}^{\beta}}\right) f_{\alpha}\right)^{-}\right]^{k} \wedge\left[\left(\sum_{s}\left(d z^{s}+\sum_{r} z^{r} \theta_{r}^{s}\right) f_{s}\right)^{-}\right]^{n-k} \\
& +n \theta_{n+1}^{n+1} f_{n+1} \wedge\left[k !\left(\theta_{n+1}^{1}-\sum_{r, \beta} z^{r} \bar{b}_{r \beta}^{\overline{1}} \bar{\theta}_{n+1}^{\beta}\right)\right. \\
& \wedge \cdots \wedge\left(\theta_{n+1}^{k}-\sum_{r, \beta} z^{r} \bar{b}_{\bar{\tau} \dot{\beta}}^{k} \bar{\theta}_{n+1}^{\beta}\right) f_{1} \\
& \wedge \cdots \wedge f_{k}(n-k-1) ! \sum_{s}\left(d z^{k+1}+\sum_{r} z^{r} \theta_{r}^{k+1}\right) \\
& \wedge \cdots \wedge\left(d z^{s}+\sum_{r} z^{r} \theta_{r}^{s}\right) \wedge \cdots \wedge\left(d z^{n}+\sum_{r} z^{r} \theta_{r}^{n}\right) f_{k+1} \\
& \left.\wedge \cdots \wedge \bar{f}_{s} \wedge \cdots \wedge f_{n}\right] \\
& \wedge\left(\begin{array}{l}
n \\
k
\end{array}\right)\left[\left(\sum_{\alpha}\left(\theta_{n+1}^{\alpha}-\sum_{r, \beta} z^{r} \bar{b}_{\bar{r} \beta}^{\tilde{\alpha}} \bar{\theta}_{n+1}^{\beta}\right) f_{\alpha}\right)^{-}\right]^{k} \\
& \wedge\left[\left(\sum_{s}\left(d z^{s}+\sum_{r} z^{r} \theta_{r}^{s}\right) f_{s}\right)^{-}\right]^{n-k} \\
& +\left(\begin{array}{l}
n \\
k
\end{array}\right)\left[\sum_{\alpha}\left(\theta_{n+1}^{\alpha}-\sum_{r, \beta} z^{r} \bar{b}_{\bar{\tau} \tilde{\beta}}^{\bar{\alpha}} \bar{\theta}_{n+1}^{\beta}\right) f_{\alpha}\right]^{k} \wedge\left[\sum_{s}\left(d z^{s}+\sum_{r} z^{r} \theta_{r}^{s}\right) f_{s}\right]^{n-k} \\
& \wedge\left[k !\left(\theta_{n+1}^{1}-\sum_{r, \beta} z^{r} \bar{b}_{\bar{r} \overline{1}}^{\overline{1}} \bar{\theta}_{n+1}^{\beta}\right) \wedge \cdots \wedge\left(\theta_{n+1}^{k}-\sum_{r, \beta} z^{r} \bar{b}_{\tilde{r} \beta}^{k} \bar{\theta}_{n+1}^{\beta}\right) f_{1}\right. \\
& \wedge \cdots \wedge f_{k}(n-k-1) ! \sum_{s}\left(d z^{k+1}+\sum_{r} z^{r} \theta_{r}^{k+1}\right) \\
& \wedge \cdots \wedge\left(d z^{s}+\sum_{r} z^{r} \theta_{r}^{s}\right) \\
& \left.\wedge \cdots \wedge\left(d z^{n}+\sum_{r} z^{r} \theta_{r}^{n}\right)\left(f_{k+1} \wedge \cdots \wedge \hat{f}_{s} \wedge \cdots \wedge f_{n}\right)\right]^{-} \\
& \left.\wedge n\left(\theta_{n+1}^{n+1} f_{n+1}\right)-\right\}
\end{aligned}
$$


This results from a straightforward calculation in which we use $y \wedge y=0$ and $\bar{y} \wedge \bar{y}=0$; in expanding $[d(y /|y|)]^{n}$ and $[d(\bar{y} /|y|)]^{n}$, we use the binomial theorem, the multiplication here being commutative. Notice that within the pair of braces above only three summands appear. Indeed, the missing summands vanish because they either have more than one $\theta_{n+1}^{n+1}$ in them, or more than one $\bar{\theta}_{n+1}^{n+1}$ in them, or more than $k f_{\alpha}$ 's, or more than $k \bar{f}_{\alpha}$ 's, or more than $(n-k) f_{r}$ 's, or more than $(n-k) \bar{f}_{r}$ 's, or else they are of the form (or its conjugate)

$$
\begin{gathered}
n \theta_{n+1}^{n+1} f_{n+1} \wedge\left[(k-1) ! \sum_{\alpha}\left(\theta_{n+1}^{1}-\sum_{r, \beta} z^{r} \bar{b}_{\bar{r} \beta}^{\overline{1}} \bar{\theta}_{n+1}^{\beta}\right)\right. \\
\wedge \cdots \wedge\left(\theta_{n+1}^{\alpha}-\sum_{r, \beta} z^{r} \bar{b}_{\bar{r} \beta}^{\bar{\alpha}} \bar{\theta}_{n+1}^{\beta}\right) \\
\left.\wedge \cdots \wedge\left(\theta_{n+1}^{k}-\sum_{r, \beta} z^{r} \bar{b}_{\bar{r} \bar{\beta}}^{\bar{\alpha} \bar{\theta}} \bar{\theta}_{n+1}^{\beta}\right)\right] f_{1} \\
\wedge \cdots \wedge \bar{f}_{\alpha} \wedge \cdots \wedge f_{n}(n-k) !\left(d z^{k+1}+\sum_{r} z^{r} \theta_{r}^{k+1}\right) \\
\wedge \cdots \wedge\left(d z^{n}+\sum_{r} z^{r} \theta_{r}^{n}\right) f_{k+1} \wedge \cdots \wedge f_{n} \wedge \text { (other forms), }
\end{gathered}
$$

whose exterior product with the factor $y=f_{n+1}+\sum z^{r} f_{r}$ (resp., with the factor $\left.\bar{y}=\bar{f}_{n+1}+\sum \bar{z}^{r}\left(f_{r}\right)^{-}\right)$is zero. Furthermore, at one point we also use $\theta_{n+1}^{n+1} \wedge \bar{\theta}_{n+1}^{n+1}$ $=0$ since $\theta_{n+1}^{n+1}=-\theta_{n+1}^{n+1}$.

Also notice that

$$
\begin{aligned}
{\left[\sum_{\alpha}\left(\theta_{n+1}^{\alpha}-\sum_{r, \beta} z^{r} \bar{b}_{\tilde{r} \beta}^{\bar{\alpha}} \bar{\theta}_{n+1}^{\beta}\right) f_{\alpha}\right]^{k} \wedge\left[\left(\sum_{\alpha}\left(\theta_{n+1}^{\alpha}-\sum_{r, \beta} z^{r} \bar{b}_{\bar{r} \bar{\beta}}^{\bar{\alpha}} \bar{\theta}_{n+1}^{\beta}\right) f_{\alpha}\right)^{-}\right]^{k} } & \\
=(k !)^{2} D \theta_{n+1}^{1} & \wedge \cdots \wedge \theta_{n+1}^{k} \wedge \bar{\theta}_{n+1}^{1} \\
& \wedge \cdots \wedge \bar{\theta}_{n+1}^{k} f_{1} \wedge \cdots \wedge f_{k} \wedge \bar{f}_{1} \wedge \cdots \wedge \bar{f}_{k}
\end{aligned}
$$

where $D$ is the $(2 k) \times(2 k)$ determinant

$$
\operatorname{det}\left(\begin{array}{cc}
I_{k} & B \\
\bar{B} & I_{k}
\end{array}\right)
$$

where $I_{k}$ is the $k \times k$ identity matrix and $B=\left(-\sum_{r} z^{r} \bar{b}_{\bar{r} \beta}^{\bar{\alpha}}\right)_{1 \leqq \alpha, \beta \leqq k}$. Since $b_{\tilde{r} \beta}^{\bar{\alpha}}=b_{\tilde{r}_{\alpha}^{\beta}}^{\bar{\beta}}$ on $F, B$ is a symmetric matrix. Hence, $D$ is the determinant of a hermitian matrix and is consequently real. 
Hence,

$[d(y /|y|)]^{n} \wedge[d(\bar{y} /|y|)]^{n} \wedge y /|y| \wedge \bar{y} /|y|$

$$
\begin{aligned}
=\frac{y \wedge \bar{y}}{|y|^{2 n+2}}(k !)^{2} D \theta_{n+1}^{1} \wedge \cdots \wedge \theta_{n+1}^{k} \wedge \theta_{n+1}^{1} \wedge \cdots \wedge \theta_{n+1}^{k} f_{1} \wedge \cdots \wedge f_{k} \\
\wedge \bar{f}_{1} \wedge \cdots \wedge \bar{f}_{k} \\
\wedge\left\{\left(\begin{array}{l}
n \\
k
\end{array}\right)^{2}\left[\sum_{s}\left(d z^{s}+\sum^{r} \theta_{r}^{s}\right) f_{s}\right]^{n-k} \wedge\left[\left(\sum_{s}\left(d z^{s}+\sum_{r} z^{r} \theta_{r}^{s}\right) f_{s}\right)^{-}\right]^{n-k}\right. \\
+n \theta_{n+1}^{n+1} f_{n+1} \wedge(n-k-1) ! \sum_{s}\left(d z^{k+1}+\sum_{r} z^{r} \theta_{r}^{k+1}\right) \\
\wedge \cdots \wedge\left(d z^{s}+\sum_{r} z^{r} \theta_{r}^{s}\right)^{\wedge} \wedge \cdots \wedge\left(d z^{n}+\sum_{r} z^{r} \theta_{r}^{n}\right) f_{k+1} \wedge \cdots \wedge \hat{f}_{s} \\
\wedge \cdots \wedge f_{n} \wedge\left(\begin{array}{l}
n \\
k
\end{array}\right)\left[\left(\sum_{s}\left(d z^{s}+\sum_{r} z^{r} \theta_{r}^{s}\right) f_{s}\right)^{-}\right]^{n-k} \\
+n\left(\theta_{n+1}^{n+1} f_{n+1}\right)^{-} \wedge(n-k-1) ! \sum_{s}\left(d z^{k+1}+\sum_{r} z^{r} \theta_{r}^{k+1}\right)^{-} \\
\wedge \cdots \wedge\left(\left(d z^{s}+\sum_{r} z^{r} \theta_{r}^{s}\right)^{-}\right)^{\wedge} \\
\wedge \cdots \wedge\left(d z^{n}+\sum_{r} z^{r} \theta_{r}^{n}\right)^{-}\left(f_{k+1} \wedge \cdots \wedge \hat{f}_{s} \wedge \cdots \wedge f_{n}\right)^{-} \\
\cdot \\
\left.\quad \wedge\left(\begin{array}{l}
n \\
k
\end{array}\right)\left[\sum_{s}\left(d z^{s}+\sum_{r} z^{r} \theta_{r}^{s}\right)\right]^{n-k}\right\} .
\end{aligned}
$$

Of course, $[d(y /|y|)]^{n} \wedge[d(\bar{y} /|y|)]^{n} \wedge y /|y| \wedge \bar{y} /|y|$ is a form on $F \times B^{m}(\tan \rho)$, but let $\lambda$ be a $C^{\infty}$ local cross section of $F$ over an open subset $U$ of $M$ and consider the map $\lambda \times$ id: $U \times B^{m}(\tan \rho) \rightarrow F \times B^{m}(\tan \rho)$. Then the form

$$
\begin{aligned}
(\lambda \times \mathrm{id}) *\left\{[d(y /|y|)]^{n}\right. & \left.\wedge[d(\bar{y} /|y|)]^{n} \wedge y /|y| \wedge \bar{y} /|y|\right\} \\
= & (\lambda \times \mathrm{id})^{*} \varphi^{*} \nu^{*} j^{*}\left[(d y)^{n} \wedge(d \bar{y})^{n} \wedge y \wedge \bar{y}\right]
\end{aligned}
$$

is an element of

$$
\left\{\Lambda^{2 n}\left[\left(T_{R}\left(U \times B^{m}(\tan \rho)\right) \otimes_{R} C\right)^{*}\right]\right\} \otimes \Lambda^{2 n+2}\left[\left.T_{R}\left(C^{n+1}\right)\right|_{0} \otimes_{R} C\right] .
$$

It follows from the remark after Proposition 5.3 that $\lambda^{*} \theta_{s}^{r}, \lambda^{*} \bar{\theta}_{s}^{r}, \lambda^{*} \theta_{n+1}^{n+1}$, and $\lambda^{*} \bar{\theta}_{n+1}^{n+1}$ all depend on the $\lambda^{*} \theta_{n+1}^{\alpha}, \lambda^{*} \bar{\theta}_{n+1}^{\alpha}$ at each point of $U$. Hence,

$(\lambda \times \mathrm{id}) *\left\{[d(y /|y|)]^{n} \wedge[d(\bar{y} /|y|)]^{n} \wedge y /|y| \wedge \bar{y} /|y|\right\}$

$$
\begin{aligned}
= & \frac{y \wedge \bar{y}}{|y|^{2 n+2}}(k !)^{2} D \circ(\lambda \times \mathrm{id}) \lambda^{*}\left(\theta_{n+1}^{1} \wedge \cdots \wedge \theta_{n+1}^{k} \wedge \bar{\theta}_{n+1}^{1} \wedge \cdots \wedge \dot{\theta}_{n+1}^{k}\right) f_{1} \\
& \wedge \cdots \wedge f_{k} \wedge \bar{f}_{1} \wedge \cdots \wedge \bar{f}_{k}\left(\begin{array}{l}
n \\
k
\end{array}\right)^{2}\left(\sum_{s} d z^{s} f_{s}\right)^{n-k} \wedge\left(\sum_{s}\left(d z^{s} f_{s}\right)^{-}\right)^{n-k}
\end{aligned}
$$


Here $y$ and $f_{j}$ mean $y \circ \varphi \circ(\lambda \times \mathrm{id})$ and $f_{j} \circ \lambda$, respectively. Now,

$$
\begin{aligned}
y \wedge\left(\sum_{s} d z^{s} f_{s}\right)^{n-k} & =(n-k) !\left(f_{n+1}+\sum_{r} z^{r} f_{r}\right) \wedge d z^{k+1} \wedge \cdots \wedge d z^{n} f_{k+1} \wedge \cdots \wedge f_{n} \\
& =(-1)^{n-k}(n-k) ! d z^{k+1} \wedge \cdots \wedge d z^{n} f_{k+1} \wedge \cdots \wedge f_{n+1}
\end{aligned}
$$

Hence,

$(\lambda \times \mathrm{id}) *\left\{[d(y /|y|)]^{n} \wedge[d(\bar{y} /|y|)]^{n} \wedge y /|y| \wedge \bar{y} /|y|\right\}$

$$
\begin{aligned}
= & (n !)^{2}(-1)^{n-k}(-1)^{k(n+1-k)} \\
& \cdot \frac{D \circ(\lambda \times \mathrm{id})}{|y|^{2 n+2}} \lambda^{*}\left(\theta_{n+1}^{1} \wedge \cdots \wedge \theta_{n+1}^{k} \wedge \bar{\theta}_{n+1}^{1} \wedge \cdots \wedge \bar{\theta}_{n+1}^{k}\right) \\
& \left.\wedge d z^{k+1} \wedge \cdots \wedge d z^{n} \wedge d \bar{z}^{k+1} \wedge \cdots \wedge d \bar{z}^{n}\right)^{-} f_{1} \\
& \wedge \cdots \wedge f_{n+1} \wedge \bar{f}_{1} \wedge \cdots \wedge \bar{f}_{n+1} .
\end{aligned}
$$

Roughly put, what we have just done is to calculate

$$
[d(y /|y|)]^{n} \wedge[d(\bar{y} /|y|)]^{n} \wedge y /|y| \wedge \bar{y} /|y|,
$$

where $y=f_{n+1}+\sum z^{r} f_{r}, O f_{1} \cdots f_{n+1}$ being unitary frames in $C^{n+1}$. Now we will again calculate the same form, this time letting $y /|y|=e_{n+1}$, where $e_{n+1}$ is the last vector of a unitary frame $O e_{1} \cdots e_{n+1}$.

Shrinking $U$ if we need to, we can find an open subset $W$ of $B^{m}(\tan \rho)$ such that $U(n+1)$ has a $C^{\infty}$ local cross section $\alpha$ over an open subset of $S^{2 n+1}$ containing $\nu \varphi(\lambda \times \mathrm{id})(U \times W)$. For $x \in U$ and $\left(z^{k+1}, \ldots, z^{n}\right) \in W$, and with

$$
O e_{1} \cdots e_{n+1}=\alpha \nu \varphi(\lambda \times \mathrm{id})(x,(z)),
$$

the equation

$$
(j \nu \varphi(\lambda \times \mathrm{id}))^{*} d y=\sum\left[(\alpha \nu \varphi(\lambda \times \mathrm{id}))^{*} \theta_{n+1}^{A}\right] e_{A}
$$

is the precise version of the statement " $d(y /|y|)=\sum \theta_{n+1}^{\prime A} e_{A}$, where $O e_{1} \cdots e_{n+1}$ is a unitary frame having $e_{n+1}=y /|y|$ with $y=f_{n+1}+\sum z^{r} f_{r}$, where $O f_{1} \cdots f_{n+1}$ $=\lambda(x)$." Letting $\beta=\alpha \nu \varphi(\lambda \times$ id $)$, we have

$$
\begin{aligned}
(j \nu \varphi(\lambda \times \mathrm{id}))^{*}\left[(d y)^{n} \wedge(d \bar{y})^{n} \wedge y \wedge \bar{y}\right] & \\
= & (-1)^{n}(n !)^{2} \beta^{*}\left(\theta_{n+1}^{\prime 1} \wedge \cdots \wedge \theta_{n+1}^{\prime n} \wedge \bar{\theta}_{n+1}^{\prime 1} \wedge \cdots \wedge \bar{\theta}_{n+1}^{\prime n}\right) e_{1} \\
& \wedge \cdots \wedge e_{n+1} \wedge \bar{e}_{1} \wedge \cdots \wedge \bar{e}_{n+1} .
\end{aligned}
$$

But

$$
\begin{aligned}
(j \nu \varphi(\lambda \times \mathrm{id}))^{*}\left[(d y)^{n}\right. & \left.\wedge(d \bar{y})^{n} \wedge y \wedge \bar{y}\right] \\
= & (\lambda \times \mathrm{id})^{*}\left\{[d(y /|y|)]^{n} \wedge[d(\bar{y} /|y|)]^{n} \wedge y /|y| \wedge \bar{y} /|y|\right\}
\end{aligned}
$$

and we have already calculated the right side of this equation in equation (7.1). Our two calculations must agree. 
Setting the right-hand members of equations (7.1) and (7.2) equal to each other and using the equation

$$
f_{1} \wedge \cdots \wedge f_{n+1} \wedge \bar{f}_{1} \wedge \cdots \wedge \bar{f}_{n+1}=e_{1} \wedge \cdots \wedge e_{n+1} \wedge \bar{e}_{1} \wedge \cdots \wedge \bar{e}_{n+1},
$$

we get the equality between absolute forms

$$
\mid \frac{D \circ(\lambda \times \mathrm{id})}{|y|^{2 n+2}} \lambda^{*}\left(\theta_{n+1}^{1} \wedge \bar{\theta}_{n+1}^{1} \wedge \cdots \wedge \theta_{n+1}^{k} \wedge \bar{\theta}_{n+1}^{k}\right) d z^{k+1} \wedge \cdots \wedge d z^{n}
$$

$$
\begin{array}{r}
\wedge d \bar{z}^{k+1} \wedge \cdots \wedge d \bar{z}^{n} \mid \\
=\left|\beta^{*}\left(\theta_{n+1}^{\prime 1} \wedge \bar{\theta}_{n+1}^{\prime 1} \wedge \cdots \wedge \theta_{n+1}^{\prime n} \wedge \tilde{\theta}_{n+1}^{\prime n}\right)\right|
\end{array}
$$

on $U \times W \subseteq M \times B^{m}(\tan \rho)$.

Notice that $\left(\frac{1}{2}\right)^{k}\left|\lambda^{*}\left(\theta_{n+1}^{1} \wedge \bar{\theta}_{n+1}^{1} \wedge \cdots \wedge \theta_{n+1}^{k} \wedge \bar{\theta}_{n+1}^{k}\right)\right|$ is the volume element of $M$ on $U$ and that $\left(\frac{1}{2}\right)^{n-k}\left|d z^{k+1} \wedge d \bar{z}^{k+1} \wedge \cdots \wedge d z^{n} \wedge d \bar{z}^{n}\right|$ is the volume element of $C^{m}$. Also recall from $\S 6$ that to $\lambda$ is associated the diffeomorphism $\lambda^{\prime}=$ $\pi \varphi(\lambda \times$ id $)$ where $\pi: C^{n+1}-\{0\} \rightarrow \boldsymbol{P}^{n}(\boldsymbol{C})$. Then $\beta \circ\left(\lambda^{\prime}\right)^{-1}$ is a $C^{\infty}$ local cross section of $U(n+1)$ over the open subset $\lambda^{\prime}(U \times W) \subseteq \boldsymbol{P}^{n}(\boldsymbol{C})$ and so

$$
\left(\frac{1}{2}\right)^{n}\left|\left(\left(\lambda^{\prime}\right)^{-1}\right)^{*} \beta^{*}\left(\theta_{n+1}^{\prime 1} \wedge \bar{\theta}_{n+1}^{\prime 1} \wedge \cdots \wedge \theta_{n+1}^{\prime n} \wedge \bar{\theta}_{n+1}^{\prime n}\right)\right|
$$

is the volume element of $\boldsymbol{P}^{n}(\boldsymbol{C})$ on $\lambda^{\prime}(U \times W) \subseteq M(\rho)$. By the way, we now see that the right side of equation (7.3) never vanishes, hence that $D$ never vanishes on $(\lambda \times \mathrm{id})(U \times W)$, and hence that $D$ never vanishes on $F \times B^{m}(\tan \rho)$ since for various $\lambda$ 's, $U$ 's, and $W$ 's, the $(\lambda \times \mathrm{id})(U \times W)$ 's cover $F \times B^{m}(\tan \rho)$. Since $D$ is real-valued and equals 1 at any point of $F \times 0, D$ must be everywhere positive.

Finally, if we write $d v$ for the volume element of $\boldsymbol{P}^{n}(\boldsymbol{C}), d v_{M}$ for that of $M$, and $d v_{C}$ for that of $C^{m}$ and if we let $p_{1}$ and $p_{2}$ be the projections of $M \times B^{m}(\tan \rho)$ onto its first and second factors, respectively, then equation (7.3) can be rewritten as

$$
\left(\lambda^{\prime}\right)^{*} d v=\frac{D \circ(\lambda \times \mathrm{id})}{|y \circ(\lambda \times \mathrm{id})|^{2 n+2}} p_{1}^{*} d v_{M} \wedge p_{2}^{*} d v_{C}
$$

on $U \times W$.

8. Calculation of the volume of a tube. For $l \geqq 1$ let $\Theta_{l}$ be the volume element of the unit $l$-sphere $S^{l}$. Consider $k^{2}$ indeterminate vectors

$$
e_{\beta}^{\alpha}=\left(e_{k+1, \beta}^{\alpha}, \ldots, e_{n \beta}^{\alpha}\right), \quad 1 \leqq \alpha, \beta \leqq k,
$$

and their conjugate vectors

$$
\left(e_{\beta}^{\alpha}\right)^{-}=\left(\left(e_{k+1, \beta}^{\alpha}\right)^{-}, \ldots,\left(e_{n \beta}^{\alpha}\right)^{-}\right), \quad 1 \leqq \alpha, \beta \leqq k .
$$

Imitating the definition of $D$, define $\tilde{D}$ as the $(2 k) \times(2 k)$ determinant

$$
\operatorname{det}\left(\begin{array}{cc}
I_{k} & E \\
\bar{E} & I_{k}
\end{array}\right)
$$


where $I_{k}$ is the $k \times k$ identity matrix and $E=\left(-\sum z^{r} e_{r \beta}^{\alpha}\right)_{1 \leqq \alpha, \beta} \leqq k$. For $1 \leqq f \leqq k$ and $1 \leqq \gamma_{1}, \ldots, \gamma_{f}, \delta_{1}, \ldots, \delta_{f} \leqq k$, define

$$
D_{\delta_{1} \cdots \delta_{f}}^{\gamma_{1} \cdots y_{f}}=\int_{S^{2 m-1}}\left|\operatorname{det}\left(\begin{array}{cc}
-\sum z^{r} e_{r \delta_{1}}^{\gamma_{1}} \cdots-\sum z^{r} e_{r \delta_{f}}^{\gamma_{1}} \\
\vdots & \vdots \\
-\sum z^{r} e_{r \delta_{1}}^{\gamma_{f}} \cdots-\sum z^{\gamma} e_{r \delta_{f}}^{\gamma_{f}}
\end{array}\right)\right|^{2} \Theta_{2 m-1} .
$$

Notice $D_{\delta_{1} \cdots \delta_{f}}^{\gamma_{1} \cdots \gamma_{f}}=0$ if two $\gamma$ 's or two $\delta$ 's coincide.

LEMMA 8.1.

$$
\int_{S^{2 m-1}} \tilde{D} \Theta_{2 m-1}=\sum_{f=0}^{k}(-1)^{f} \sum_{\alpha_{1}<\cdots<\alpha_{f}: \beta_{1}<\cdots<\beta_{f}} D_{\beta_{1} \cdots \beta_{f}}^{\alpha_{1} \cdots \alpha_{f},}
$$

the term for $f=0$ being understood to equal 1 .

Proof. Consider any $(2 k) \times(2 k)$ matrix

$$
\left(m_{q}^{p}\right)=\left(\begin{array}{ll}
I_{k} & A \\
C & I_{k}
\end{array}\right)
$$

where $I_{k}$ is the $k \times k$ identity matrix and where $A$ and $C$ are $k \times k$ matrices

$$
A=\left(a_{\delta}^{\gamma}\right)_{1 \leqq \gamma, \delta \leqq k}, \quad C=\left(c_{\delta}^{\gamma}\right)_{1 \leqq \gamma, \delta \leqq k} .
$$

The determinant of $\left(m_{q}^{p}\right)$ is a sum of $(2 k)$ ! terms $\pm m_{q_{1}}^{1} \cdots m_{q_{2 k}}^{2 k}$. Any such term is zero if it contains among its factors $m_{q_{l}}^{l}$ a number of 1's from the upper left quarter of $\left(m_{q}^{p}\right)$ that differs from the number of 1's it contains from the lower right quarter. The remaining terms are of the form $s a_{\alpha\left(\alpha_{1}\right)}^{\alpha_{1}} \cdots a_{\alpha\left(\alpha_{f}\right)}^{\alpha_{f}} c_{\gamma\left(\gamma_{1}\right)}^{\gamma_{1}} \cdots c_{\gamma\left(\gamma_{f}\right)}^{\gamma_{f}}$, where $\alpha_{1}<\cdots<\alpha_{f}, \gamma_{1}<\cdots<\gamma_{f}$, where $\alpha\left(\alpha_{1}\right), \ldots, \alpha\left(\alpha_{f}\right)$ is a permutation of $\gamma_{1}, \ldots, \gamma_{f}$, where $\gamma\left(\gamma_{1}\right), \ldots, \gamma\left(\gamma_{f}\right)$ is a permutation of $\alpha_{1}, \ldots, \alpha_{f}$, and where $s$ is \pm 1 . In such a term let $s_{1}$ be the sign (i.e., \pm 1 ) of $\alpha\left(\alpha_{1}\right) \cdots \alpha\left(\alpha_{f}\right)$, and let $s_{2}$ be the sign of $\gamma\left(\gamma_{1}\right) \cdots \gamma\left(\gamma_{f}\right)$. In order to write the numbers $k+\alpha\left(\alpha_{1}\right), \ldots, k+\alpha\left(\alpha_{f}\right), \gamma\left(\gamma_{1}\right), \ldots, \gamma\left(\gamma_{f}\right)$ in increasing order, we have to perform a permutation of sign $s_{1}$ on the first $f$ numbers and a permutation of sign $s_{2}$ on the last $f$ numbers, and then we must perform the permutation of sign $(-1)^{f}$ which interchanges the new 1 st and $(f+1)$ th numbers, the new 2nd and $(f+2)$ th numbers, etc. Hence, $s=s_{1} s_{2}(-1)^{f}$. Therefore,

$$
\operatorname{det}\left(m_{q}^{p}\right)=\sum_{f=0}^{k}(-1)^{f} \sum_{\alpha_{1}<\cdots<\alpha_{f} ; \gamma_{1}<\cdots<\gamma_{f}} \operatorname{det}\left(\begin{array}{cc}
a_{\gamma_{1}}^{\alpha_{1}} \cdots a_{\gamma_{f}}^{\alpha_{1}} \\
\vdots & \vdots
\end{array}\right) \operatorname{det}\left(\begin{array}{cc}
c_{\alpha_{1}}^{\gamma_{1}} \cdots c_{\alpha_{f}}^{\gamma_{1}} \\
\vdots & \vdots
\end{array}\right) \text {. }
$$

The lemma now follows. Q.E.D.

The unitary group $U(m)$ acts as isometries on $S^{2 m-1}$ and hence preserves the volume element. It then follows from the definition of $D_{\delta_{1} \cdots \delta_{f}}^{\gamma_{1} \cdots \gamma_{f}}$ that $D_{\delta_{1} \cdots \delta_{f}}^{\gamma_{1} \cdots \gamma_{f}}$ is invariant under the action of $U(m)$ on the vectors $e$. Hence, by the first main theorem on unitary invariants $\left[2\right.$, p. 97], $D_{\delta_{1} \ldots \delta_{f}}^{\gamma_{1} \ldots y_{f}}$ is a polynomial in the inner products

$$
E_{\gamma_{c} \delta_{b}}^{\gamma_{a} \delta_{d}^{\delta}}=\sum_{r} e_{r \delta_{b}}^{\gamma_{a}}\left(e_{r \delta_{d}}^{\gamma_{c}}\right)^{-}, \quad 1 \leqq a, b, c, d \leqq f
$$


But since $D_{b_{1} \ldots \delta_{f}}^{\gamma_{1} \ldots y_{f}}$ is a linear combination of monomials

$$
e_{r_{1} \delta_{\rho(1)}}^{\gamma_{\sigma(1)}} \cdots e_{r_{f} \delta_{\rho(f)}}^{\gamma_{\sigma(f)}}\left(e_{r_{f+1} \delta_{v(1)}}^{\gamma_{\mu(1)}} \cdots e_{r_{2 f} \delta_{v(f)}}^{\gamma_{\mu(f)}}\right)^{-}
$$

where $\sigma, \rho, \mu$, and $\nu$ are elements of $S(f)$, the group of permutations on $f$ letters, then $D_{\delta_{1} \cdots \delta_{f}}^{\gamma_{1} \cdots \gamma_{f}}$ must actually be a linear combination of terms

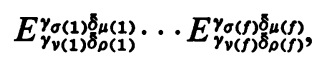

for $\sigma, \rho, \mu, \nu \in S(f)$. Also notice that the integrand in the right side of equation (8.1) is the product of two determinants and that $D_{\delta_{1} \cdots \delta_{f}}^{\gamma_{1} \cdots y_{f}}$ is skew-symmetric in the rows and in the columns of each determinant. Therefore, $D_{\delta_{1} \ldots \delta_{f}}^{\gamma_{1} \ldots \gamma_{f}}$ must be a multiple of

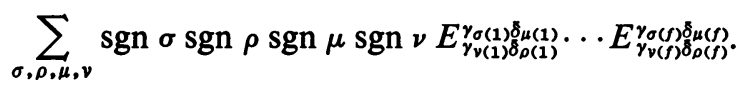

In particular, for $\alpha_{1}<\cdots<\alpha_{f}$ and $\beta_{1}<\cdots<\beta_{f}, D_{\beta_{1} \cdots \beta_{f}}^{\alpha_{1} \cdots \alpha_{f}}$ is a multiple of

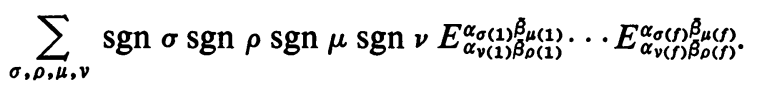

To evaluate this multiple, we specialize the indeterminate vectors $e$ by the equations

$$
e_{\beta_{b}}^{\alpha_{a}}=\left(\delta_{b}^{a}, 0, \ldots, 0\right), \quad 1 \leqq a, b \leqq f
$$

Then

$$
\begin{aligned}
E_{\alpha_{\nu(b)} \beta_{\rho(b)}}^{\alpha_{\alpha}(b) \beta_{\mu(b)}} & =1 & & \text { if } \sigma(b)=\rho(b) \text { and } \mu(b)=\nu(b), \\
& =0 & & \text { otherwise. }
\end{aligned}
$$

Hence, with the above specialization we have

$$
\sum_{\sigma, \rho, \mu, v} \operatorname{sgn} \sigma \operatorname{sgn} \rho \operatorname{sgn} \mu \operatorname{sgn} \nu E_{\alpha_{v(1) \beta \rho(1)}}^{\alpha_{\sigma(1)} \bar{\beta}_{\mu(1)}} \cdots E_{\alpha_{v(f) \beta \rho(f)}}^{\alpha_{\sigma(f)} \beta_{\mu(f)}}=(f !)^{2} .
$$

On the other hand, with the specialization of equation (8.2),

$$
D_{\beta_{1} \cdots \beta_{f}}^{\alpha_{1} \cdots \alpha_{f}}=\int_{\left(z^{k+1}, \cdots, z^{n}\right) \in S^{2 m-1}}\left|z^{k+1}\right|^{2 f} \Theta_{2 m-1} .
$$

To evaluate this integral, let us define, for $l \geqq 1$, the subset $H_{+}^{l}$ of $S^{l}$ by

$$
H_{+}^{l}=\left\{\left(t_{1}, \ldots, t_{l+1}\right) \in S^{l}: \text { all } t \text { 's are positive }\right\}
$$

let us define the subset $H^{l}$ of $S^{l}$ by

$$
H^{l}=\left\{\left(t_{1}, \ldots, t_{l+1}\right) \in S^{l}: \text { all } t \text { 's are nonnegative }\right\} ;
$$

and let us define the subset $\tilde{S}^{2 m-1}$ of $S^{2 m-1}$ by

$$
\widetilde{S}^{2 m-1}=\left\{\left(z_{1}, \ldots, z_{m}\right) \in S^{2 m-1}: \text { no } z \text { is } 0\right\} .
$$

Write the elements of $C^{m}$ as $\left(z^{k+1}, \ldots, z^{n}\right)$ and consider the natural diffeomorphism $\eta: \widetilde{S}^{2 m-1} \rightarrow H_{+}^{m-1} \times S^{1} \times \cdots \times S^{1}$, with $m$ factors $S^{1}$, defined by

$$
\eta\left(z^{k+1}, \ldots, z^{n}\right)=\left(\left(t^{k+1}, \ldots, t^{n}\right), e^{i \theta_{k+1}}, \ldots, e^{i \theta_{n}}\right)
$$


if $z^{r}=e^{i \theta_{r} t^{r}}, t^{r}>0$. Let $S_{r}^{1}$ be the $(r-k)$ th factor $S^{1}$ in $H_{+}^{m-1} \times S^{1} \times \cdots \times S^{1}$, and let $d \theta_{r}$ be the distance element on $S_{r}^{1}$. Consider the absolute form

$$
\Theta_{m-1} \wedge t^{k+1} d \theta_{k+1} \wedge \cdots \wedge t^{n} d \theta_{n}
$$

on $H_{+}^{m-1} \times S^{1} \times \cdots \times S^{1}$.

Lemma 8.2. $\eta^{*}\left(\Theta_{m-1} \wedge t^{k+1} d \theta_{k+1} \wedge \cdots \wedge t^{n} d \theta_{n}\right)=\Theta_{2 m-1} \cdot$.

Proof. The idea of the proof is as follows: If $O f_{1} \cdots f_{m}$ is an orthonormal frame field in $\boldsymbol{R}^{m}$, then

$$
\Theta_{m-1}=\left|\left(d f_{m}, f_{1}\right) \cdots\left(d f_{m}, f_{m-1}\right)\right| .
$$

Let us write $f_{r-k}=\left(t_{r-k}^{k+1}, \ldots, t_{r-k}^{n}\right), k+1 \leqq r \leqq n$. If we define

$$
\begin{aligned}
e_{1}= & \left(-\sin \theta_{k+1}, \cos \theta_{k+1}, 0, \ldots, 0\right), \\
e_{3}= & \left(0,0,-\sin \theta_{k+2}, \cos \theta_{k+2}, 0, \ldots, 0\right), \\
\ldots \ldots \ldots \ldots & \\
e_{2 m-1}= & \left(0, \ldots, 0,-\sin \theta_{n}, \cos \theta_{n}\right),
\end{aligned}
$$

and, for $k+1 \leqq r \leqq n$,

$$
e_{2(r-k)}=\left(t_{r-k}^{k+1} \cos \theta_{k+1}, t_{r-k}^{k+1} \sin \theta_{k+1}, \ldots, t_{r-k}^{n} \cos \theta_{n}, t_{r-k}^{n} \sin \theta_{n}\right),
$$

then

$$
\Theta_{2 m-1}=\left|\left(d e_{2 m}, e_{1}\right) \cdots\left(d e_{2 m}, e_{2 m-1}\right)\right| .
$$

With the use of the equations

$$
\begin{aligned}
\left(d e_{2 m}, e_{2(r-k)-1}\right) & =\eta^{*}\left(t^{r} d \theta_{r}\right), \\
\left(d e_{2 m}, e_{2(r-k)}\right) & =\eta^{*}\left(d f_{m}, f_{r-k}\right)+\text { forms in the } \eta^{*} d \theta^{\prime} \mathrm{s},
\end{aligned}
$$

a computation of wedge products does it. Q.E.D.

Returning to equation (8.4) and writing

$$
\left(z^{k+1}, \ldots, z^{n}\right)=\left(t^{k+1} e^{i \theta_{k+1}}, \ldots, t^{n} e^{i \theta_{n}}\right),
$$

where $t^{r}>0$, we have

$$
\begin{aligned}
\int_{\left(z^{k+1}, \ldots, z^{n}\right) \in S^{2 m-1}}\left|z^{k+1}\right|^{2 f} \Theta_{2 m-1} & \\
& =\int_{H_{+}^{m-1}}\left(t^{k+1}\right)^{2 f+1} t^{k+2} \cdots t^{n}\left[\int_{S^{1} \times \cdots \times S^{1}} d \theta_{k+1} \wedge \cdots \wedge d \theta_{n}\right] \Theta_{m-1} \\
& =(2 \pi)^{m} \int_{H^{m-1}}\left(t^{k+1}\right)^{2 f+1} t^{k+2} \cdots t^{n} \Theta_{m-1} .
\end{aligned}
$$

To calculate this last integral we prove the following lemma.

\section{LEMMA 8.3.}

$$
\int_{\left(t_{1}, \ldots, t_{l}\right) \in H^{l-1}} t_{1}^{k_{1}} \cdots t_{l}^{k_{l}} \Theta_{l-1}=2\left(\Gamma\left(\frac{1}{2}\left(\sum_{p=1}^{l} k_{p}+l\right)\right)\right)^{-1} 2^{-l} \prod_{p=1}^{l} \Gamma\left(\frac{k_{p}+1}{2}\right) .
$$


Proof. On the one hand,

$\int_{0}^{\infty} \cdots \int_{0}^{\infty} x_{1}^{k_{1}} \cdots x_{l}^{k_{l}} e^{-x_{1}^{2}-\cdots-x_{l}^{2}} d x_{1} \cdots d x_{l}=\prod_{p=1}^{l} \int_{0}^{\infty} x_{p}^{k_{p}} e^{-x_{p}^{2}} d x_{p}=\prod_{p=1}^{l} \frac{1}{2} \Gamma\left(\frac{k_{p}+1}{2}\right)$.

On the other hand, if we write $x=r t$ for $t \in H^{l-1}$ and $r \geqq 0$, then

$$
\begin{aligned}
\int_{0}^{\infty} \cdots \int_{0}^{\infty} x_{1}^{k_{1}} \cdots x_{l}^{k_{l}} & e^{-x_{1}^{2}-\cdots-x_{l}^{2}} d x_{1} \cdots d x_{l} \\
& =\int_{0}^{\infty} r^{\Sigma} e^{-r^{2}} d r \int_{H^{l-1}} t_{1}^{k_{1}} \cdots t_{l}^{k_{l}} \Theta_{l-1}, \quad \text { where } \Sigma=\sum k^{p}+l-1, \\
& =\frac{1}{2} \Gamma\left(\frac{1}{2}\left(\sum k_{p}+l\right)\right) \int_{H^{l-1}} t_{1}^{k_{1}} \cdots t_{1}^{k_{l} \Theta_{l-1} .}
\end{aligned}
$$

The lemma now follows. Q.E.D.

Consequently, for the specialization of equation (8.2)

$$
D_{\beta_{1} \cdots \beta_{f}}^{\alpha_{1} \cdots \alpha_{f}}=2 \pi^{m} \frac{f !}{(f+m-1) !}
$$

From equations (8.3) and (8.5) it follows that we have the equation in indeterminates $e$

(8.6) $\quad D_{\beta_{1} \cdots \beta_{f}}^{\alpha_{1} \cdots \alpha_{f}}=\frac{2 \pi^{m}}{f !(f+m-1) !} \sum_{\sigma, \rho, \mu, v} \operatorname{sgn} \sigma \operatorname{sgn} \rho \operatorname{sgn} \mu \operatorname{sgn} \nu E_{\alpha_{v(1)} \beta_{\rho(1)}}^{\alpha_{\sigma(1)} \beta_{\mu(1)}} \cdots E_{\alpha_{v(f)} \beta_{\rho(f)}}^{\alpha_{\sigma(f)} \beta_{\mu(f)}}$

where $\alpha_{1}<\cdots<\alpha_{f}$ and $\beta_{1}<\cdots<\beta_{f}$, the $\alpha$ 's and $\beta$ 's being taken from the integers $1, \ldots, k$. Since both sides of equation (8.6) are unchanged when two $\alpha$ 's or two $\beta$ 's are interchanged, and since both sides vanish when two or more $\alpha$ 's coincide or when two or more $\beta$ 's coincide,

$$
\begin{aligned}
& \sum_{\alpha_{1}<\cdots<\alpha_{f}: \beta_{1}<\cdots<\beta_{f}} D_{\beta_{1} \cdots \beta_{f}}^{\alpha_{1} \cdots \alpha_{f}}=\frac{1}{(f !)^{2}} \sum_{1 \leqq \alpha_{a}, \beta_{b} \leqq k ; 1 \leqq a, b \leqq f} D_{\beta_{1} \cdots \beta_{f}}^{\alpha_{1} \cdots \alpha_{f}} \\
& =\frac{2 \pi^{m}}{(f !)^{3}(f+m-1) !} \sum_{\sigma, \rho, \mu, \nu} \operatorname{sgn} \sigma \operatorname{sgn} \rho \operatorname{sgn} \mu \operatorname{sgn} \nu \\
& \sum_{1 \leqq \alpha_{a}, \beta_{b} \leqq k: 1 \leqq a, b \leqq f} E_{\alpha_{v(1) \beta_{\rho(1)}}}^{\alpha_{\sigma(1)} \tilde{\beta}_{\mu(1)}} \cdots E_{\alpha_{v(f)} \beta_{\rho(f)}}^{\alpha_{\sigma(f)} \tilde{\beta}_{\mu(f)}} \\
& =\frac{2 \pi^{m}}{f !(f+m-1) !} \sum \delta\left(\begin{array}{l}
\alpha_{1} \cdots \alpha_{f} \\
\gamma_{1} \cdots \gamma_{f}
\end{array}\right) \delta\left(\begin{array}{l}
\beta_{1} \cdots \beta_{f} \\
\delta_{1} \cdots \delta_{f}
\end{array}\right) E_{\gamma_{1} \delta_{1}}^{\alpha_{1} \bar{\beta}_{1}} \cdots E_{\gamma_{f} \delta f}^{\alpha_{f} \tilde{\delta}_{f}},
\end{aligned}
$$

where in this last summation the $\alpha$ 's, $\beta$ 's, $\gamma$ 's, and $\delta$ 's run from 1 to $k$ and where, in general, $\delta\left({ }_{\mu_{1} \cdots \mu_{f}}^{\varepsilon_{1} \cdots \varepsilon_{f}}\right)$ is 1 if $\varepsilon_{1}, \ldots, \varepsilon_{f}$ are distinct and an even permutation of $\mu_{1}, \ldots, \mu_{f}$, is -1 if $\varepsilon_{1}, \ldots, \varepsilon_{f}$ are distinct and an odd permutation of $\mu_{1}, \ldots, \mu_{f}$, and is 0 otherwise.

Now we specialize the indeterminate $e$ 's by letting

$$
e_{r \delta}^{\gamma}=\bar{b}_{r \delta}^{\bar{\gamma}}, \quad 1 \leqq \gamma, \delta \leqq k, k+1 \leqq r \leqq n .
$$


If $\gamma$ is a $C^{\infty}$ local cross section of $F$ over an open subset of $U\left(\boldsymbol{P}^{n}(C), M\right)$, then by equation (5.5) $b_{\bar{r} \bar{\alpha}}^{\bar{\alpha}} \circ \gamma=h_{\bar{r} \beta}^{\tilde{\alpha}}$ and

$$
E_{\delta \bar{\varepsilon}}^{\alpha \tilde{\beta}} \circ \gamma=\sum \bar{h}_{\bar{r} \bar{\alpha}}^{\bar{\alpha}} h_{\bar{r} \delta}^{\bar{\beta}}=-H_{\delta \bar{\varepsilon}}^{\alpha \tilde{\beta}},
$$

where $H_{\delta \bar{\varepsilon}}^{\alpha \beta}$ is the excess holomorphic curvature tensor. The summation

$$
H_{2 f}=\sum_{1 \leqq \alpha, \beta, \gamma, \delta \leqq k} \delta\left(\begin{array}{l}
\alpha_{1} \cdots \alpha_{f} \\
\gamma_{1} \cdots \gamma_{f}
\end{array}\right) \delta\left(\begin{array}{l}
\beta_{1} \cdots \beta_{f} \\
\delta_{1} \cdots \delta_{f}
\end{array}\right) H_{\gamma_{1} \delta_{1}}^{\alpha_{1} \beta_{1}} \cdots H_{\gamma_{f} \delta f}^{\alpha_{f} \beta_{f}}
$$

on $U\left(\boldsymbol{P}^{n}(\boldsymbol{C}), M\right)$ is constant on fibers over points of $M$ (and hence is a function $H_{2 f}$ on $\left.M\right)$ since $H_{\delta \bar{\varepsilon}}^{\alpha \bar{\beta}}$ transforms along fibers like a tensor. We understand $H_{0}$ to

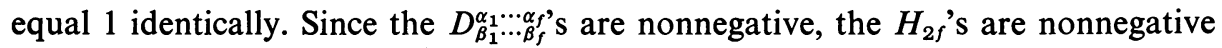
for even $f$, nonpositive for odd $f$. Taking note of Lemma 8.1, we see we have proved the following proposition.

Proposition 8.4. If $\lambda$ is a $C^{\infty}$ local cross section of $F$ over an open subset $U$ of $M$, then

$$
\int_{S^{2 m-1}} D \circ \lambda \Theta_{2 m-1}=\sum_{f=0}^{k} c_{f} H_{2 f}
$$

on $U$, where $c_{f}=2 \pi^{m} / f !(f+m-1) !$.

Now we can finish finding the volume of the tube $M(\rho)$. Since $M$ is compact, we can take a finite open cover $\left\{U_{\xi}\right\}$ of $M$ such that over each $U_{\xi}, F$ has a $C^{\infty}$ local cross section $\lambda_{\xi}$ and such that there exists a $C^{\infty}$ partition of unity $\left\{f_{\xi}\right\}$ on $M$ subordinate to $\left\{U_{\xi}\right\}$. Let

$$
\lambda_{\xi}^{\prime}: U_{\xi} \times B^{m}(\tan \rho) \rightarrow \exp \left(\bigcup_{x \in U_{\xi}} B_{x}(\rho)\right)
$$

be the diffeomorphism associated to $\lambda_{\xi}$; so

$$
\lambda_{\xi}^{\prime}\left(x,\left(z^{r}\right)\right)=\left[f_{n+1}+\sum z^{r} f_{r}\right]
$$

where $O f_{1} \cdots f_{n+1}=\lambda_{\xi}(x)$. Let $p_{1}, p_{2}$ be the projections of $M \times B^{m}(\tan \rho)$ onto the first and second factors, respectively. The open cover $\left\{\lambda_{\xi}^{\prime}\left(U_{\xi} \times B^{m}(\tan \rho)\right)\right\}$ of $M(\rho)$ is also finite, and $\left\{f_{\xi} \circ p_{1} \circ \lambda_{\xi}^{\prime-1}\right\}$ is a $C^{\infty}$ partition of unity subordinate to this cover. Let $q_{1}, q_{2}$ be the projections of $S^{2 m-1} \times(0, \tan \rho)$ onto its first and second factors, respectively. Let $h$ be the diffeomorphism of $B^{m}(\tan \rho)-\{0\}$ onto $S^{2 m-1} \times(0, \tan \rho)$ which sends $\left(z^{k+1}, \ldots, z^{n}\right)$ to

$$
\left(\frac{\left(z^{k+1}, \ldots, z^{n}\right)}{\left|\left(z^{k+1}, \ldots, z^{n}\right)\right|},\left|\left(z^{k+1}, \ldots, z^{n}\right)\right|\right) \text {. }
$$

In what follows, $y \circ\left(\lambda_{\xi} \times \mathrm{id}\right)\left(x,\left(z^{r}\right)\right)$ means $\varphi \circ\left(\lambda_{\xi} \times \mathrm{id}\right)\left(x,\left(z^{r}\right)\right)=f_{n+1}+\sum z^{r} f_{r}$, where $O f_{1} \cdots f_{n+1}=\lambda_{\xi}(x)$. As in $\S 7, d v, d v_{M}$, and $d v_{C}$ will be the volume elements of $\boldsymbol{P}^{n}(\boldsymbol{C})$, $M$, and $C^{m}$, respectively. All exterior forms in the following are to be considered as absolute forms. 
The volume of the tube $M(\rho)$ is then

$$
\begin{aligned}
\int_{M(\rho)} d v & =\sum_{\xi} \int_{\lambda_{\xi}^{\prime}\left(U_{\xi} \times B^{m}(\tan \rho)\right)}\left(f_{\xi} \circ p_{1} \circ \lambda_{\xi}^{\prime-1}\right) d v \\
& =\sum_{\xi} \int_{U_{\xi} \times B^{m}(\tan \rho)}\left(f_{\xi} \circ p_{1}\right) \frac{D \circ\left(\lambda_{\xi} \times \mathrm{id}\right)}{\left|y \circ\left(\lambda_{\xi} \times \mathrm{id}\right)\right|^{2 n+2}} p_{1}^{*} d v_{M} \wedge p_{2}^{*} d v_{C} \\
& =\sum_{\xi} \int_{U_{\xi}} f_{\xi}\left\{\int_{S^{m-1} \times(0, \tan \rho)} \frac{D \circ\left(\lambda_{\xi} \times \mathrm{id}\right)}{\left|y \circ\left(\lambda_{\xi} \times \mathrm{id}\right) \circ h^{-1}\right|^{2 n+2}} \cdot q_{1}^{*} \Theta_{2 m-1} \wedge q_{2}^{*}\left(r^{2 m-1} d r\right)\right\} d v_{M} \\
& =\sum_{\xi} \int_{U_{\xi}} f_{\xi}\left\{\int_{0}^{\tan \rho} \frac{r^{2 m-1}}{\left(1+r^{2}\right)^{n+1}}\left[\int_{S^{2 m-1}} D \circ\left(\lambda_{\xi} \times \mathrm{id}\right) \circ h^{-1} \Theta_{2 m-1}\right] d r\right\} d v_{M} \\
& =\sum_{\xi} \int_{U_{\xi}} f_{\xi}\left\{\int_{0}^{\tan \rho} \frac{r^{2 m-1}}{\left(1+r^{2}\right)^{n+1}}\left[\sum_{f=0}^{k} c_{f} r^{2 f} H_{2 f}\right] d r\right\} d v_{M} \\
& =\sum_{f=0}^{k} c_{f}\left[\int_{M} H_{2 f} d v_{M}\right] J_{f}(\rho),
\end{aligned}
$$

where

$$
\begin{aligned}
J_{f}(\rho) & =\int_{0}^{\tan \rho} \frac{r^{2 m+2 f-1}}{\left(1+r^{2}\right)^{n+1}} d r \\
& =\int_{0}^{\rho}(\sin s)^{2 m+2 f-1}(\cos s)^{2 k-2 f+1} d s .
\end{aligned}
$$

In the second equation in the above sequence of equations, equation (7.4) is used. In the third equation from the end we have used the fact that if $r>0$, then

$$
\int_{s^{2 m-1}}\left|\operatorname{det}\left(\begin{array}{c}
-r \sum z^{s} \bar{b}_{s \beta_{1}}^{\bar{\alpha}_{1}}\left(\lambda_{\xi}\right) \cdots \\
\vdots \\
-r \sum z^{s} \bar{b}_{s \beta_{1}}^{\alpha_{f}}\left(\lambda_{\xi}\right) \ldots
\end{array}\right)\right|^{2} \Theta_{2 m-1}=r^{2 f} D_{\beta_{1} \ldots \beta_{f}^{\alpha_{1}} \ldots \lambda_{\xi}}^{\alpha_{1} \ldots}
$$

This completes the derivation of our formula.

If we take for $M$ a $\boldsymbol{P}^{k}(C)$ imbedded in $\boldsymbol{P}^{n}(C), k<n$, the volume of the tube of radius $\pi / 2$ about $\boldsymbol{P}^{k}(C)$ should equal the volume of $\boldsymbol{P}^{n}(C)$, which is $\pi^{n} / n !$.

LEMMA 8.5.

$$
\int_{0}^{\infty} \frac{r^{2 m-1}}{\left(1+r^{2}\right)^{n+1}} d r=\frac{k !(m-1) !}{2 n !}
$$

where $m=n-k$ and where $k$ and $n$ are integers satisfying $n>k \geqq 0$.

Proof. Let $L(n, k)$ be the statement of the lemma for integers $n, k . L(k+1, k)$ is easily checked to hold. Moreover, integration by parts shows that $L(n, k)$ implies $L(n+1, k)$. Hence, the lemma follows. Q.E.D. 
Now, $H_{\gamma \delta}^{\alpha \beta}$ vanishes identically on $\boldsymbol{P}^{k}(\boldsymbol{C}) \subseteq \boldsymbol{P}^{n}(\boldsymbol{C})$ since the holomorphic curvature tensor of $\boldsymbol{P}^{n}(\boldsymbol{C})$, with all indices no greater than $k$, equals the holomorphic curvature tensor of $\boldsymbol{P}^{k}(\boldsymbol{C})$. Hence, for $\boldsymbol{P}^{k}(\boldsymbol{C})$ all $H_{2 f}$ 's vanish except when $f=0$. Thus, by our formula for the volume of a tube, the volume of $\boldsymbol{P}^{n}(C)$ equals

$$
\begin{aligned}
c_{0} \operatorname{vol}\left(\boldsymbol{P}^{k}(\boldsymbol{C})\right) J_{0}(\pi / 2) & =\frac{2 \pi^{m}}{(m-1) !} \operatorname{vol}\left(\boldsymbol{P}^{k}(\boldsymbol{C})\right) \int_{0}^{\infty} \frac{r^{2 m-1}}{\left(1+r^{2}\right)^{n+1}} d r \\
& =\frac{2 \pi^{m}}{(m-1) !} \frac{\pi^{k}}{k !} \frac{k !(m-1) !}{2 n !}=\frac{\pi^{n}}{n !}
\end{aligned}
$$

where we have used the fact that the volume of $\boldsymbol{P}^{k}(C)$ is $\pi^{k} / k !$.

For a complex submanifold $M$ of complex dimension $k$,

$$
H_{2}=\sum\left[R_{\alpha \beta}^{\alpha \beta}-\left(\delta_{\alpha \beta} \delta_{\alpha \beta}+\delta_{\alpha \alpha} \delta_{\beta \beta}\right)\right]=R-k-k^{2},
$$

where $R$, the holomorphic scalar curvature of $M$, equals half the riemannian scalar curvature. When $k=1$, then $J_{0}(\rho)=(\sin \rho)^{2 n-2} /(2 n-2)-(\sin \rho)^{2 n} / 2 n$ and $J_{1}(\rho)$ $=(\sin \rho)^{2 n} / 2 n$, so the volume of the tube of radius $\rho$ about a nonsingular complex curve $M$ is

$$
\frac{2 \pi^{n}}{(n-1) !}(\sin \rho)^{2 n-2}\left[\left(1-\frac{n+1}{n} \sin ^{2} \rho\right) d+\frac{\sin ^{2} \rho}{n}(1-g)\right],
$$

and where $d$ is the degree and $g$ is the genus of the curve and where we have used the Gauss-Bonnet formula and the formula vol $M=2 \pi d[2$, p. 120].

\section{BIBLIOGRAPHY}

1. R. L. Bishop and R. J. Crittenden, Geometry of manifolds, Pure and Appl. Math., vol. 15, Academic Press, New York, 1964. MR 29 \#6401.

2. S.-S. Chern, Characteristic classes of Hermitian manifolds, Ann. of Math. (2) 47 (1946), 85-121. MR 7, 470.

3. H. Flanders, Development of an extended exterior differential calculus, Trans. Amer. Math. Soc. 75 (1953), 311-326. MR 15, 161.

4. S. Helgason, Differential geometry and symmetric spaces, Pure and Appl. Math., vol. 12, Academic Press, New York, 1962. MR 26 \#2986.

5. H. Weyl, On the volume of tubes, Amer. J. Math. 61 (1939), 461-472.

UNiversity of SAN Francisco,

San Francisco, California 94117 